\title{
Analysis of servo-constraint problems for underactuated multibody systems
}

\author{
R. Seifried ${ }^{1}$ and W. Blajer ${ }^{2}$ \\ ${ }^{1}$ Institute of Engineering and Computational Mechanics, University of Stuttgart, Pfaffenwaldring 9 , \\ 70569 Stuttgart, Germany \\ ${ }^{2}$ Faculty of Mechanical Engineering, Technical University of Radom, ul. Krasickiego 54, \\ 26-600 Radom, Poland
}

Correspondence to: R. Seifried (robert.seifried@itm.uni-stuttgart.de)

Received: 5 October 2012 - Revised: 4 January 2013 - Accepted: 11 January 2013 - Published: 19 February 2013

\begin{abstract}
Underactuated multibody systems have fewer control inputs than degrees of freedom. In trajectory tracking control of such systems an accurate and efficient feedforward control is often necessary. For multibody systems feedforward control by model inversion can be designed using servo-constraints. So far servo-constraints have been mostly applied to differentially flat underactuated mechanical systems. Differentially flat systems can be inverted purely by algebraic manipulations and using a finite number of differentiations of the desired output trajectory. However, such algebraic solutions are often hard to find and therefore the servo-constraint approach provides an efficient and practical solution method. Recently first results on servo-constraint problems of non-flat underactuated multibody systems have been reported. Hereby additional dynamics arise, so-called internal dynamics, yielding a dynamical system as inverse model. In this paper the servo-constraint problem is analyzed for both, differentially flat and non-flat systems. Different arising important phenomena are demonstrated using two illustrative examples. Also strategies for the numerical solution of servo-constraint problems are discussed.
\end{abstract}

\section{Introduction}

Multibody systems with fewer control inputs than degrees of freedom are called underactuated. Typical examples are multibody systems with passive joints, body flexibility, joint elasticities, aircrafts and cranes. A possible performance task of such systems is output trajectory tracking, e.g. tracking of the end-effector point of flexible manipulators. In order to obtain a good performance in trajectory tracking an accurate and efficient feedforward control is often necessary, which then can be combined with a feedback controller. A feedforward control is an inverse model of the multibody system, providing the necessary control inputs for exact output reproduction. Depending on the system's properties the inverse model might be purely algebraic or might contain a dynamical part. While there is a large amount of various linear and nonlinear feedback control strategies available, there exist much less concepts for feedforward control design of nonlinear systems.
A very appealing and efficient feedforward control design approach for multibody systems is the use of socalled servo-constraints, which are also called programm constraints or control constraints, see Blajer (1992); Campbell (1995); Kirgetov (1967); Rosen (1999); Bajodah et al. (2005). Thereby the equations of motion of the underactuated multibody system are supplemented by a servo-constraint, which enforces the exact reproduction of the desired output trajectory. This yields as set of differential-algebraic equations (DAEs), whose solution provides the searched control input. Due to some similarities to classical constraints, servoconstraint problems have recently been attracted increasing attention in the multibody system dynamics context.

The servo-constraint approach is often applied to socalled differentially flat underactuated mechanical systems, such as cranes, aircrafts and mass-spring chains, as investigated in Betsch et al. (2008); Blajer and Kolodziejczyk (2004, 2007, 2011); Fumagalli et al. (2011). The theory 
of differential flatness is a differential algebraic approach, which is due to the fundamental work of Fliess et al. (1995). Differential flatness is a structural property, which is determined by the system and the imposed system output. Roughly speaking, in a differentially flat system a system output can be found, from which all states and inputs can be determined without integration. However, a finite number of derivatives of the output might have to be taken. Differentially flat nonlinear systems can be seen as a generalization of linear controllable systems, as discussed by Rothfuss et al. (1997). These systems have the favorable property that they can be inverted purely by algebraic manipulations and using a finite number of differentiations of the system output. However, such algebraic solutions are often hard to find and therefore the servo-constraint approach provides an efficient and practical solution method for model inversion. An extension of this servo-constraint approach is its use in feedback linearization, where the model is formulated in redundant coordinates, see Frye and Fabien (2011).

More recently also first results on the application of servoconstraints to non-flat systems, such as, e.g. flexible manipulators or systems with passive joints, have been reported, see Seifried (2012a); Moberg and Hanssen (2007); Kovács et al. (2011); Masarati et al. (2011). In the inverse model of non-flat systems additional dynamics arise, so-called internal dynamics. Thus the inverse model is a dynamical model. This internal dynamics of the inverse model might be stable or unstable. Therefore it must be analyzed carefully in order to obtain a meaningful solution and is treated extensively in differential-geometric nonlinear control theory, see Isidori (1995); Sastry (1999).

In this paper the servo-constraint problem is analyzed for both, flat and non-flat systems. Two approaches for analyzing and solving the servo-constraint problem are taken. These are a projection approach and a coordinate transformation approach. The projection approach is due to Blajer and Kolodziejczyk (2004, 2007). It allows a straightforward formulation of the servo-constraint problem and simplifies significantly the numerical solution of the arising DAEs. This method has also been applied to differentially flat multibody systems with mixed geometric and servo-constraints as reported by Betsch et al. (2008) and Blajer and Kolodziejczyk (2011). In the coordinate transformation approach the servoconstraint problem is reformulated in new coordinates containing the output. In this way a DAE formulation might be avoided, which significantly simplifies the analysis of the servo-constraint system dynamics. The equivalence of both approaches is discussed in Seifried (2012a). In this paper a slightly different formulation of the coordinate transformation approach is used, see also Blajer and Seifried (2012); Blajer et al. (2013). Based on both formulations of the servoconstraint problem, the various possible situations which can occur in servo-constraint problems are demonstrated. Therefore two illustrative examples are used. These are a massspring-damper system on a car and a rotational manipulator arm with passive joint. Finally some remarks on the numerical solution of servo-constraint problems are given. The numerical solution methods depend strongly on the previously analyzed system properties.

\section{Trajectory tracking of underactuated multibody systems}

Multibody systems with $f$ degrees of freedom and $m$ control inputs are considered. For underactuated multibody systems it is imperative $m<f$. The kinematics of multibody systems is described using generalized coordinates $\boldsymbol{q} \in \mathbb{R}^{f}$. The control inputs $\boldsymbol{u} \in \mathbb{R}^{m}$ are assumed to be control forces and torques. Based on d'Alembert's principle the equations of motion in minimal form can be derived using the NewtonEuler-Formalism, see e.g. Schiehlen et al. (2006). The nonlinear equations of motion are given by,

$M(q) \ddot{q}+f(q, \dot{q})=B(q) u$,

where $\boldsymbol{M} \in \mathbb{R}^{f \times f}$ is the symmetrical and positive definite mass matrix and $\boldsymbol{f} \in \mathbb{R}^{f}$ summarizes all generalized forces. These generalized forces are given by $\boldsymbol{f}=\boldsymbol{k}-\boldsymbol{g}$, whereby $\boldsymbol{k}$ is the vector of generalized gyroscopic, centrifugal and Coriolis forces and $g$ are all applied forces such as gravity. The system inputs $\boldsymbol{u} \in \mathbb{R}^{m}$ are distributed by the input matrix $\boldsymbol{B} \in \mathbb{R}^{f \times m}$ on the directions of the generalized coordinates. Generally it is assumed that there is no redundant actuation and thus the rank of $\boldsymbol{B}$ equals $m$. It is often useful to partition the equations of motion of underactuated multibody systems in actuated and unactuated parts,

$$
\left[\begin{array}{ll}
\boldsymbol{M}_{\mathrm{aa}}(\boldsymbol{q}) & \boldsymbol{M}_{\mathrm{au}}(\boldsymbol{q}) \\
\boldsymbol{M}_{\mathrm{au}}^{T}(\boldsymbol{q}) & \boldsymbol{M}_{\mathrm{uu}}(\boldsymbol{q})
\end{array}\right]\left[\begin{array}{l}
\ddot{\boldsymbol{q}}_{\mathrm{a}} \\
\ddot{\boldsymbol{q}}_{\mathrm{u}}
\end{array}\right]+\left[\begin{array}{l}
\boldsymbol{f}_{\mathrm{a}}(\boldsymbol{q}, \dot{\boldsymbol{q}}) \\
\boldsymbol{f}_{\mathrm{u}}(\boldsymbol{q}, \dot{\boldsymbol{q}})
\end{array}\right]=\left[\begin{array}{c}
\boldsymbol{B}_{\mathrm{a}}(\boldsymbol{q}) \\
\boldsymbol{B}_{\mathrm{u}}(\boldsymbol{q})
\end{array}\right] \boldsymbol{u} .
$$

Thereby $\boldsymbol{q}_{\mathrm{a}} \in \mathbb{R}^{m}$ are the actuated generalized coordinates and $\boldsymbol{q}_{\mathrm{u}} \in \mathbb{R}^{f-m}$ are the unactuated coordinates. This partition is based on the requirement that the rank of the submatrix $\boldsymbol{B}_{\mathrm{a}} \in \mathbb{R}^{m \times m}$ equals $m$. In many instances, e.g. passive joint manipulators or flexible multibody systems, the input submatrices might reduce to $\boldsymbol{B}_{\mathrm{a}}=\boldsymbol{I}$ and $\boldsymbol{B}_{\mathrm{u}}=\mathbf{0}$, where $\boldsymbol{I}$ is the identity matrix. In this case each generalized coordinate of $\boldsymbol{q}_{\mathrm{a}}$ is directly collocated with one component of input $\boldsymbol{u}$.

\subsection{Output trajectory tracking control}

The control task which is considered in this paper is output trajectory tracking. Thereby, a system output $\boldsymbol{y} \in \mathbb{R}^{m}$ of the multibody system is given by

$y=h(q)$.

This system output can depend linearly or nonlinearly on all generalized coordinates. A typical example for such a system output is an end-effector position of a manipulator. The dimension of input and output coincide, an assumption often 


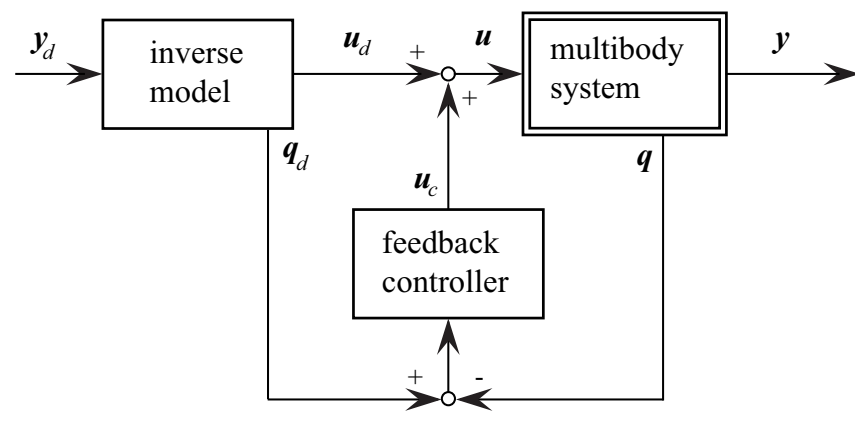

Figure 1. Control structure with feedforward and feedback controller.

required in nonlinear control theory. The velocity and acceleration of the system output follow as

$$
\begin{aligned}
\dot{\boldsymbol{y}} & =\boldsymbol{H}(\boldsymbol{q}) \dot{\boldsymbol{q}}, \\
\ddot{\boldsymbol{y}} & =\boldsymbol{H}(\boldsymbol{q}) \ddot{\boldsymbol{q}}+\overline{\boldsymbol{h}}(\boldsymbol{q}, \dot{\boldsymbol{q}}) .
\end{aligned}
$$

Thereby $\boldsymbol{H} \in \mathbb{R}^{m \times f}$ is the Jacobian matrix of the system output and $\overline{\boldsymbol{h}}=\dot{\boldsymbol{H}} \dot{\boldsymbol{q}} \in \mathbb{R}^{m}$. In trajectory tracking the system output (Eq. 3) should track exactly a time-variant output trajectory $\boldsymbol{y}_{d}(t)$, which is defined in space and time. Thus also velocity $\dot{\boldsymbol{y}}_{d}(t)$ and acceleration $\ddot{\boldsymbol{y}}_{d}(t)$ of the system output are specified.

Multibody systems in output trajectory tracking perform often large working motion. Thus, the equations of motion (Eq. 1) are highly nonlinear and in many instances linear control theory cannot be applied. An efficient approach of output trajectory tracking of nonlinear systems is a so-called two design degree-of-freedom control structure, consisting of a feedforward control and an additional feedback control, see Fig. 1. Thereby the feedforward control is an inverse model of the multibody system. It provides for a given output trajectory $\boldsymbol{y}_{d}(t)$ the associated control inputs $\boldsymbol{u}_{d}$ and the trajectories $\boldsymbol{q}_{d}$ of all generalized coordinates. In the absence of any uncertainties and disturbances the control input $\boldsymbol{u}_{d}$ can be applied to the multibody system and reproduces the desired output trajectory exactly. Since in a real hardware implementation always some parameter uncertainties and disturbances arise, additional feedback control is necessary and provides additional control input $\boldsymbol{u}_{c}$. For feedback control design the computed trajectories $\boldsymbol{q}_{d}$ of the generalized coordinates can be used as reference signal. In trajectory tracking the most control action is provided by the feedforward part and the feedback part has to compensate only small derivations following from uncertainties and disturbances. Therefore, often simple linear control strategies such as PID control might be applicative for the feedback part.

For fully actuated multibody systems, such as fully actuated manipulators, it is $f=m$. Then, the inverse model can be derived easily by pure algebraic manipulations, see e.g. Spong et al. (2006). In this case the inverse model can be split into inverse kinematics and inverse dynamics. In inverse kinematics of a fully actuated system the nonlinear output equation (Eq. 3) can be solved, providing for given $\boldsymbol{y}_{d}$ the trajectories $\boldsymbol{q}_{d}$ of the generalized coordinates. This can be achieved by algebraic manipulations, numerical solution or differential kinematics, respectively. For details it is pointed to Siciliano et al. (2010). By using the determined $\boldsymbol{q}_{d}$ and its derivatives $\dot{\boldsymbol{q}}_{d}, \ddot{\boldsymbol{q}}_{d}$ in the equations of motion (Eq. 1) the control inputs $\boldsymbol{u}_{d}$ can be computed algebraically.

For underactuated multibody systems the inverse kinematics following from Eq. (3) is under-determined. Also the inverse dynamics problem cannot be solved since the input matrix $\boldsymbol{B}$ is not invertible. Thus, for underactuated multibody systems the splitting of the model inversion into inverse kinematics and inverse dynamics is in general not possible and both parts must be be solved concurrently. For differentially flat underactuated multibody systems a purely algebraic inverse model can be derived, using a finite number of derivatives of the system output $\boldsymbol{y}$. In contrast, for non-flat systems the inverse model is a dynamical system. Flatness is a system property determined by the system dynamics and the chosen output, but is independent of the used coordinates to describe the multibody system.

\subsection{Servo-constraints in multibody systems}

An efficient and straightforward approach for model inversion is the use of servo-constraints. The basic idea of servoconstraints is the enforcement of output trajectory tracking by introducing constraint equations. These servo-constraints can be seen as an extension of classical geometric constraints, which makes this approach so appealing in multibody system dynamics. In order to introduce the concept of servo-constraints, classical constraints are briefly reviewed. For example, consider a multibody system with a kinematic loop, see Fig. 2. In order to obtain its equation of motion the kinematic loop is cut at a suitable joint, removing $n$ constraints. Then, the corresponding equations of motion of the open chain system are derived in minimal form and the kinematic loop is enforced by introducing algebraic loop closing constraints $\boldsymbol{c}_{c}(\boldsymbol{q}, t)=\mathbf{0} \in \mathbb{R}^{n}$. Restricting to a multibody system without control action, the equations of motion of the open chain system yields together with the constraint equations,

$\boldsymbol{M}(\boldsymbol{q}) \ddot{\boldsymbol{q}}+\boldsymbol{f}(\boldsymbol{q}, \dot{\boldsymbol{q}})=\boldsymbol{C}^{T} \lambda$,

$\boldsymbol{c}_{c}(\boldsymbol{q})=\mathbf{0}$

Thereby $\boldsymbol{C}=\partial \boldsymbol{c}_{c} / \partial \boldsymbol{q} \in \mathbb{R}^{f \times n}$ is the Jacobian matrix of the constraint equation and $\lambda \in \mathbb{R}^{n}$ are the Lagrangian multipliers. These ensure that the loop closing constraints are met. Equations (6)-(7) form a set of differential algebraic equations (DAE). The numerical solution of the DAE provides the forward dynamics of the closed loop multibody system with trajectories of the states $\boldsymbol{q}, \dot{\boldsymbol{q}}$ and the Lagrangian multipliers $\lambda$. 
a.) multibody system with kineamtik loop

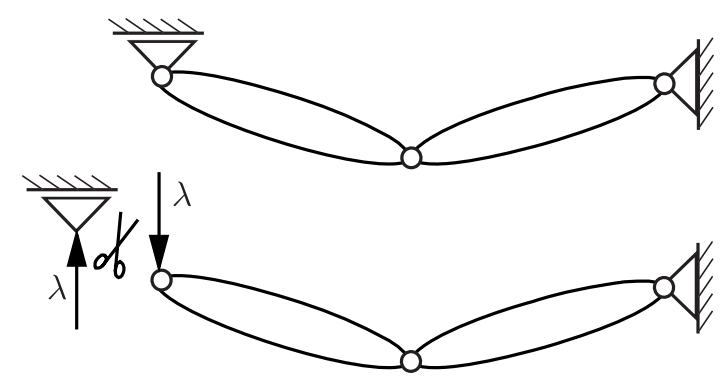

b.) multibody system with servo-constraints

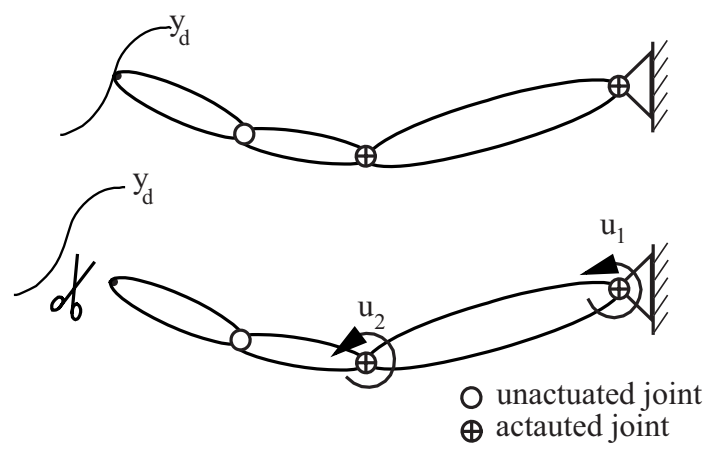

Figure 2. Multibody systems with constraints.

In order to derive an inverse model for underactuated multibody systems a similar approach can be used. The problem of tracking a desired trajectory $\boldsymbol{y}_{d}$ is induced by introducing $m$ algebraic servo-constraints. The inverse model of an underactuated multibody systems is then given by the equations motion (Eq. 1) and the servo-constraints,

$\boldsymbol{M}(\boldsymbol{q}) \ddot{\boldsymbol{q}}+\boldsymbol{f}(\boldsymbol{q}, \dot{\boldsymbol{q}})=\boldsymbol{B}(\boldsymbol{q}) \boldsymbol{u}$,

$\boldsymbol{c}(\boldsymbol{q}, t)=\boldsymbol{h}(\boldsymbol{q})-\boldsymbol{y}_{d}(t)=\mathbf{0}$,

where Eq. (9) represents the servo-constraint. As noticed by Blajer (1997a); Blajer and Kolodziejczyk (2004, 2007) the servo-constraint problem Eqs. (8)-(9) is mathematically equivalent to Eqs. (6)-(7). Thereby the desired trajectory $\boldsymbol{y}_{d}(t)$ can be interpreted as a drift in time of constraint manifold $\boldsymbol{c}(\boldsymbol{q})=\mathbf{0}$ in the system configuration space, see Blajer (2001). The generalized actuating forces $\boldsymbol{B} \boldsymbol{u}$ can then be viewed as a generalized reaction forces of the servo constraints. Thus, structurally the generalized actuation forces $\boldsymbol{B} \boldsymbol{u}$ corresponds to the generalized reaction forces $\boldsymbol{C}^{T} \lambda$. Therefore, in the servo-constraint approach the control inputs $\boldsymbol{u}$ ensure that the servo-constraints are met. The similarities between both cases are illustrated in Fig. 2 .

At first, multibody systems with servo- and physical constraints show many similarities. However, servo-constraint problems can posses more complex properties, which have to be understood to obtain a meaningful solution. For a multi- body system with classical constraints the matrix $C^{T}$ projects the Lagrange multipliers $\lambda$ on the directions orthogonal to the constraint manifold, which is defined by the constraint equation (Eq. 7). Thus, the generalized reaction forces $C^{T} \lambda$ are orthogonal to the constraint manifold, see Fig. 3. Therefore such a system is called an ideal orthogonal realization.

In contrast, the generalized actuation forces $\boldsymbol{B} \boldsymbol{u}$ are not necessarily ideal orthogonal to the constraint manifold which is defined by the servo-constraint (Eq. 9). The actuation forces $\boldsymbol{B} \boldsymbol{u}$ might be non-ideal orthogonal or in the extreme case even tangential to the constraint manifold, see Fig. 3. These cases are called non-ideal orthogonal realization and tangential realization, respectively. Thereby, ideal and nonideal orthogonal realization have many similarities, and are in the following only distinguished, if different phenomena occur. In the case of both types of orthogonal realization control inputs are explicitly available in all directions orthogonal to the servo-constraints and can directly actuate the servoconstraint condition. However, in the case of non-ideal orthogonal realization the projection with $\boldsymbol{B}$ yields also components in direction tangential to the constraint manifold. In a tangential realization the control inputs are projected in tangential direction. Then, the control inputs $\boldsymbol{u}$ cannot actuate directly the constraint condition, but output tracking of $\boldsymbol{y}_{d}$ might be still possible due to coupling with other forces of the system, see Blajer and Kolodziejczyk (2004). This tangential projection is often connected to underactuated differentially flat systems. However, as will be shown in section 3 tangential realization can also arise in non-flat systems. Further analysis from a geometric point of view are also found in Blajer (1992), Blajer and Kolodziejczyk (2004) and Blajer and Kolodziejczyk (2007).

For systems with multiple inputs and outputs it might occur that both, orthogonal (ideal or non-ideal) and tangential realization exist. Thus, in so-called mixed orthogonaltangential realizations only some outputs can be directly influenced by the inputs, while others can only be influenced indirectly. A measure of the control singularity is the deficiency in rank $p$ of the matrix

$\boldsymbol{P}=\boldsymbol{H} \boldsymbol{M}^{-1} \boldsymbol{B}$.

The case $p=m$ indicates that all components of the system output $\boldsymbol{y}$ can directly be actuated by the inputs $\boldsymbol{u}$. The case $0<p<m$ shows that only $p$ components of the output can be regulated in the orthogonal way, while realization of the other $m-p$ output components are without direct involvement of the actuating forces $\boldsymbol{B u}$. Finally, $p=0$ refers to a pure tangential realization of servo-constraints, as the system inputs do not directly influence the outputs.

If the solution of the servo constraint problem (Eqs. 8-9) exists, the numerical solution of this DAE provides the trajectories of all states $\boldsymbol{q}_{d}, \dot{\boldsymbol{q}}_{d}$ as well as the corresponding control inputs $\boldsymbol{u}_{d}$. For characterizing DAEs often the differentiation index is used. Following Hairer and Wanner (2010) the differentiation index is the number of derivatives of the DAE, 
a.) ideal orthogonal realization

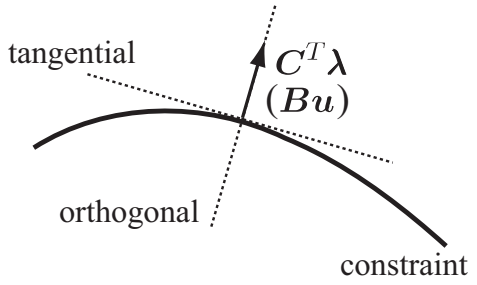

manifold

b.) non-ideal orthogonal realization

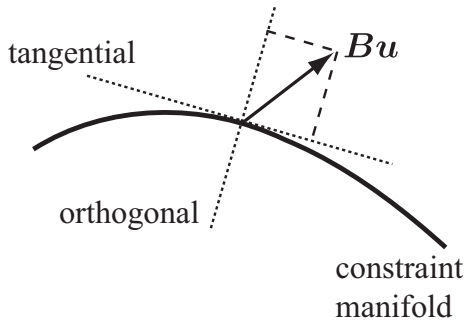

c.) tangential realization

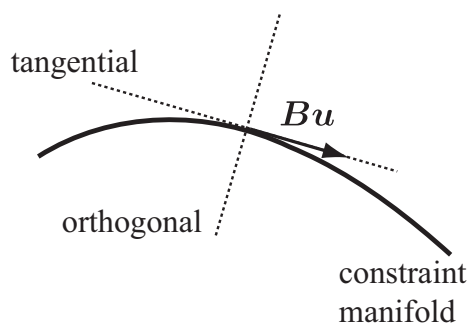

Figure 3. Possible realizations in servo-constraint problems.

or parts of it, until an ordinary differential equation (ODE) for all unknowns are obtained. For multibody systems with classical constraints it is well know that they have differentiation index 3. Here the constraint equation (Eq. 7) must be differentiated three times in order to derive an ODE for the unknown $\lambda$. This is provided by the fact that for classical constraints the matrix (Eq. 10) becomes $\boldsymbol{P}=\boldsymbol{C} \boldsymbol{M}^{-1} \boldsymbol{C}^{T}$, which has full rank if the constraints are independent, see Hairer and Wanner (2010).

In the case of servo-constraints this is not any more necessarily true. In the case of orthogonal realizations $\boldsymbol{P}$ has full rank and index 3 arises. However, if the servo-constraint problem includes a tangential realization $\boldsymbol{P}$ is singular and higher differentiation index arise. For various mechanical systems with servo-constraints the differentiation index is analyzed in Campbell (1995).

The differentiation index is closely related to the relative degree used in differential geometric nonlinear control theory. An extensive treatment of this nonlinear control theory is given in Isidori (1995); Sastry (1999). Restricting to systems with $n$ states and one input and one output, the relative degree $r$ is the number of Lie derivatives of the system output until the first time the control input occurs. If the relative degree is $r=n$, then the system is differentially flat and a purely algebraic inverse model can be extracted. In the case $r<n$ socalled internal dynamics remain and the inverse model will contain a dynamical part. For extension to systems with multiple inputs and outputs it is pointed to the aforementioned nonlinear control literature. In Campbell (1995) it is pointed out that the differentiation index is one higher than the relative degree, if the internal dynamics are not affected by a constraint.

\subsection{Projection approach}

In Blajer (1997a) it is shown that the equation of motion with additional constraints can be projected into two complementary subspaces in velocity space. These are the constrained and unconstrained subspace. The unconstrained subspace is tangential to the constraint manifold, while the constrained subspace is orthogonal to it, see Fig. 3. The constrained subspace describes in the servo-constraint context the output subspace and follows from projection with the Jacobian matrix $\boldsymbol{H} \in \mathbb{R}^{m \times f}$ of the output, which has rank $m$. For the second subspace an orthogonal complement $\boldsymbol{D} \in \mathbb{R}^{f \times f-m}$ with rank $f-m$ must be derived, such that

$\boldsymbol{H D}=\mathbf{0}$ and $\boldsymbol{D}^{T} \boldsymbol{H}^{T}=\mathbf{0}$

is satisfied. Using these two matrices the equations of motion are projected into the two subspaces,

$\left[\begin{array}{c}\boldsymbol{H} \boldsymbol{M}^{-1} \\ \boldsymbol{D}^{T}\end{array}\right](\boldsymbol{M} \ddot{\boldsymbol{q}}+\boldsymbol{f}-\boldsymbol{B u})=\mathbf{0}$

which yields,

$\boldsymbol{H} \ddot{\boldsymbol{q}}+\boldsymbol{H} \boldsymbol{M}^{-1} \boldsymbol{f}=\boldsymbol{H} \boldsymbol{M}^{-1} \boldsymbol{B} \boldsymbol{u}$,

$\boldsymbol{D}^{T} \boldsymbol{M} \ddot{\boldsymbol{q}}+\boldsymbol{D}^{T} \boldsymbol{f}=\boldsymbol{D}^{T} \boldsymbol{B u}$.

With the output equation (Eq. 5) at acceleration level the corresponding servo-constraint provides $\boldsymbol{H} \ddot{\boldsymbol{q}}=\ddot{\boldsymbol{y}}_{d}-\overline{\boldsymbol{h}}$. This relationship can be used in Eq. (13). Introducing the new state $\boldsymbol{v}=\dot{\boldsymbol{q}}$ and adding the servo-constraints at position level, after reordering the projected servo-constraint formulation, provides

$$
\begin{aligned}
\dot{\boldsymbol{q}} & =\boldsymbol{v} \\
\boldsymbol{D}^{T} \boldsymbol{M} \dot{\boldsymbol{v}} & =-\boldsymbol{D}^{T} \boldsymbol{f}+\boldsymbol{D}^{T} \boldsymbol{B u} \\
\mathbf{0} & =\boldsymbol{P} \boldsymbol{u}-\boldsymbol{H} \boldsymbol{M}^{-1} \boldsymbol{f}+\overline{\boldsymbol{h}}-\ddot{\boldsymbol{y}}_{d} \\
\mathbf{0} & =\boldsymbol{h}(\boldsymbol{q})-\boldsymbol{y}_{d} .
\end{aligned}
$$

This forms a set of $2 f+m$ differential-algebraic equations for the $2 f+m$ unknowns $\boldsymbol{q}, \boldsymbol{v}, \boldsymbol{u}$. Equation (17) has dimension $m$ and describes an algebraic equation in $\boldsymbol{q}, \boldsymbol{v}, \boldsymbol{u}$. Together with the $m$ servo-constraints (Eq. 18) there are $2 m$ algebraic equations in these DAEs. By this projection and incorporating the servo-constraint at acceleration level, an index reduction by 
two is achieved, which in general simplifies the numerical solution. For example, for the crane considered in Blajer and Kolodziejczyk $(2004,2007)$, the differentiation index is reduced from 5 to 3 using this projection approach.

\subsection{Coordinate transformation}

The numerical solution of Eqs. (15)-(18) provides the model inversion. However, for analysis purpose it might be of advantage to write first the equations of motion in a new set of coordinates containing the system output. In addition, this might also be used to simplify the projections in Eqs. (15)(18). Also for systems with orthogonal realization an inverse model as ODE can be derived in a straightforward way. For example, as new set of coordinates

$\overline{\boldsymbol{q}}=\left[\begin{array}{c}\boldsymbol{y} \\ \boldsymbol{q}_{\mathrm{u}}\end{array}\right]=\left[\begin{array}{c}\boldsymbol{h}\left(\boldsymbol{q}_{\mathrm{a}}, \boldsymbol{q}_{\mathrm{u}}\right) \\ \boldsymbol{q}_{\mathrm{u}}\end{array}\right]=\phi(\boldsymbol{q})$

might be used, which yields for the velocities

$\dot{\overline{\boldsymbol{q}}}=\left[\begin{array}{c}\dot{\boldsymbol{y}} \\ \dot{\boldsymbol{q}}_{\mathrm{u}}\end{array}\right]=\left[\begin{array}{cc}\boldsymbol{H}_{\mathrm{a}} & \boldsymbol{H}_{\mathrm{u}} \\ \mathbf{0} & \boldsymbol{I}\end{array}\right]\left[\begin{array}{c}\dot{\boldsymbol{q}}_{\mathrm{a}} \\ \dot{\boldsymbol{q}}_{\mathrm{u}}\end{array}\right]=\frac{\partial \boldsymbol{\phi}(\boldsymbol{q})}{\partial \boldsymbol{q}} \dot{\boldsymbol{q}}$.

It is noted that the first row of Eq. (20) is identical to Eq. (4), i.e. $\boldsymbol{H}=\left[\begin{array}{ll}\boldsymbol{H}_{\mathrm{a}} & \boldsymbol{H}_{\mathrm{u}}\end{array}\right]$. In order to be an admissible coordinate transformation, it must be a diffeomorphism, i.e. smooth and invertible. Relationship (Eq. 19) is at least a local diffeomorphism if the Jacobian matrix in Eq. (19) is non-singular. Inspecting Eq. (19) shows, that this is true if the submatrix $\boldsymbol{H}_{\mathrm{a}}$ is nonsingular. This requires that the output equation (Eq. 3) depends on all $m$ actuated coordinates $\boldsymbol{q}_{\mathrm{a}}$. This is for example the case for manipulators with flexible links or passive joints in end-effector tracking. A counterexample is a manipulator with flexible joints in end-effector tracking, see e.g. De Luca (1998).

With the results of Eq. (5) the coordinate transformation (Eq. 19) at acceleration level is,

$\ddot{\overline{\boldsymbol{q}}}=\left[\begin{array}{c}\ddot{\boldsymbol{y}} \\ \ddot{\boldsymbol{q}}_{\mathrm{u}}\end{array}\right]=\left[\begin{array}{cc}\boldsymbol{H}_{\mathrm{a}} & \boldsymbol{H}_{\mathrm{u}} \\ \mathbf{0} & \boldsymbol{I}\end{array}\right]\left[\begin{array}{c}\ddot{\boldsymbol{q}}_{\mathrm{a}} \\ \ddot{\boldsymbol{q}}_{\mathrm{u}}\end{array}\right]+\left[\begin{array}{c}\overline{\boldsymbol{h}} \\ \mathbf{0}\end{array}\right]$

From the equations of motion (Eq. 1) follows $\ddot{\boldsymbol{q}}=\boldsymbol{M}^{-1}(\boldsymbol{B u}-$ $f$ ) which can be inserted in Eq. (21) and yields

$$
\begin{aligned}
\ddot{\boldsymbol{y}} & =\boldsymbol{P u}-\boldsymbol{H} \boldsymbol{M}^{-1} \boldsymbol{f}+\overline{\boldsymbol{h}}, \\
\ddot{\boldsymbol{q}}_{\mathrm{u}} & =[\mathbf{0} \vdots \boldsymbol{I}] \boldsymbol{M}^{-1}(\boldsymbol{B u}-\boldsymbol{f}) .
\end{aligned}
$$

In all entries of these two equations the original states $\boldsymbol{q}_{\mathrm{a}}, \dot{\boldsymbol{q}}_{\mathrm{a}}$ must be replaced by the new states $\boldsymbol{y}, \dot{\boldsymbol{y}}$. Therefore the upper part of Eq. (19) must be solved for $\boldsymbol{q}_{\mathrm{a}}$, which is in general nonlinear. Afterwards the velocities $\dot{\boldsymbol{q}}_{\mathrm{a}}$ can be computed from the linear equations provided by Eq. (20).

The two second order differential Eqs. (22)-(23) represent the equations of motion of the multibody system expressed in the new coordinates $\overline{\boldsymbol{q}}$, which include the system output $\boldsymbol{y}$.
The equations of motion (Eqs. 22-23) can be helpful in analyzing both, orthogonal and tangential realization, as will be seen in the next subsection.

The coordinate transformation approach is inspired by differential-geometric control theory, which is the basis of feedback linearization and can also be used for feedforward control design, see Isidori (1995); Sastry (1999). Thereby, nonlinear systems are transformed by diffeomorphic coordinate transformations into the so-called nonlinear inputoutput normal form using new states, containing the output and a finite number of its time derivatives. The application of this nonlinear control theory to underactuated multibody systems in orthogonal realization is given in Seifried (2012a,b).

The following short discussion highlights some correspondence of the servo-constraint approach with aforementioned nonlinear control theory for underactuated multibody systems in orthogonal realization. In this case, the equations of motion (Eqs. 22-23) in new coordinates $\overline{\boldsymbol{q}}$ are identical to the nonlinear input-output normal form. The matrix $\boldsymbol{P}=\boldsymbol{H} \boldsymbol{M}^{-1} \boldsymbol{B}$ is called decoupling matrix in nonlinear control theory. Equation (22) links the input $\boldsymbol{u}$ to the second derivative of the output $\ddot{\boldsymbol{y}}$, describing the input-output relationship. Equation (23) is called in nonlinear control theory the internal dynamics. This is the remaining system dynamics of the inverse model. From this input-output normal form feedback linearization and feedforward control design are easily possible. For a desired output trajectory $\boldsymbol{y}_{d}$ the necessary control input follows from Eq. (22) as

$\boldsymbol{u}_{d}=\boldsymbol{P}^{-1}\left(\boldsymbol{H} \boldsymbol{M}^{-1} \boldsymbol{f}-\overline{\boldsymbol{h}}+\ddot{\boldsymbol{y}}_{d}\right)$.

It is noticed that this is structurally identical to Eq. (17), however expressed in terms of the new coordinates. Equation (24) is an algebraic expression for the input, depending solely on the known values $\boldsymbol{y}_{d}, \dot{\boldsymbol{y}}_{d}$ and the unknowns $\boldsymbol{q}_{\mathrm{u}}, \dot{\boldsymbol{q}}_{u}$. The later ones follow from solving the internal dynamics by applying Eq. (24) to the ODE Eq. (23), resulting in

$$
\begin{aligned}
\ddot{\boldsymbol{q}}_{\mathrm{u}} & =[\mathbf{0} \vdots \boldsymbol{I}] \boldsymbol{M}^{-1}\left[\boldsymbol{B} \boldsymbol{P}^{-1}\left(\boldsymbol{H} \boldsymbol{M}^{-1} \boldsymbol{f}-\overline{\boldsymbol{h}}+\ddot{\boldsymbol{y}}_{d}\right)-\boldsymbol{f}\right], \\
& =\overline{\boldsymbol{a}}\left(\boldsymbol{q}_{\mathrm{u}}, \dot{\boldsymbol{q}}_{u}, \boldsymbol{y}_{d}, \dot{\boldsymbol{y}}_{d}, \ddot{\boldsymbol{y}}_{d}\right) .
\end{aligned}
$$

These are $f-m$ second order differential equations for $\boldsymbol{q}_{\mathrm{u}}$, driven by the desired output $\boldsymbol{y}_{d}$ and its derivatives $\dot{\boldsymbol{y}}_{d}, \ddot{\boldsymbol{y}}_{d}$.

\section{Illustrative example 1: mass on car}

The different phenomena which might arise in servoconstraint problems of underactuated multibody systems are demonstrated using a spring-mass system mounted on a car, which is shown in Fig. 4. The car with mass $m_{1}$ moves along the horizontal $e_{1}$ axis and is actuated by the force $u=F$. On the car a mass $m_{2}$ is mounted, which moves along an axis which is inclined by the angle $\alpha$. The system is described by the two generalized coordinates $\boldsymbol{q}=[x, s]$, whereby $x$ is the horizontal car position and $s$ the relative position of the 


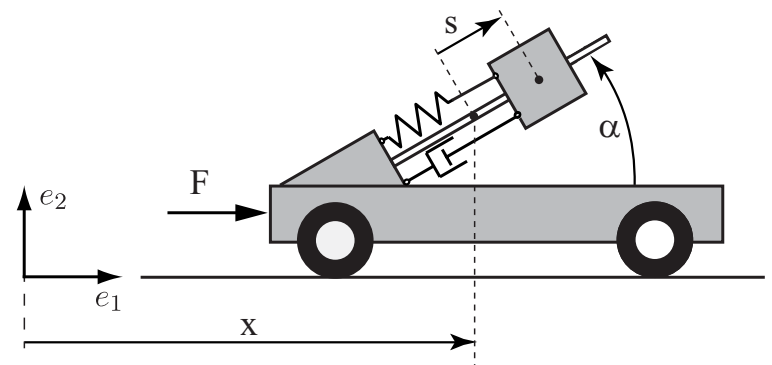

Figure 4. Mass on car system.

mass along the inclined axis. Thereby, $q_{a}=x$ is the actuated coordinate and $q_{u}=s$ is the unactuated coordinate. The mass is supported by a parallel spring-damper combination, with spring and damping coefficients $k, d$, respectively. In the equilibrium position it is $s=0$. This yields the equations of motion

$\left[\begin{array}{cc}m_{1}+m_{2} & m_{2} \cos \alpha \\ m_{2} \cos \alpha & m_{2}\end{array}\right]\left[\begin{array}{c}\ddot{x} \\ \ddot{s}\end{array}\right]+\left[\begin{array}{c}0 \\ k s+d \dot{s}\end{array}\right]=\left[\begin{array}{c}F \\ 0\end{array}\right]$.

The system output is the horizontal position of the mass,

$y=x+s \cos \alpha$

which should follow a predefined trajectory $y_{d}(t)$. This yields the servo constraint

$c(\boldsymbol{q}, t)=x+s \cos \alpha-y_{d}(t)$.

Equations (26) and (28) form the servo-constrained problem. From Eq. (28) follow the two projection matrices,

$\boldsymbol{H}=\left[\begin{array}{ll}1 & \cos \alpha\end{array}\right], \quad \boldsymbol{D}=\left[\begin{array}{c}\cos \alpha \\ -1\end{array}\right]$.

With these matrices the projected Eqs. (15)-(18) of the servoconstraint problem can be computed. Thereby it follows from Eq. (18)

$P=\boldsymbol{H} \boldsymbol{M}^{-1} \boldsymbol{B}=\frac{\sin ^{2} \alpha}{m_{1}+m_{2} \sin ^{2} \alpha}$.

It becomes apparent that this matrix is nonsingular for $\alpha \neq 0$, i.e. it poses an orthogonal realization. However for $\alpha=0$ a tangential realization occurs.

In order to analyze this servo-constraint problem in more detail, the coordinate transformation approach presented in Sect. 2.4 is applied. The new set of coordinates containing the output are chosen as $\overline{\boldsymbol{q}}=[y, s]$. The system dynamics in new coordinates follows from evaluating Eqs. (22)-(23),

$$
\begin{gathered}
\ddot{y}=-\frac{m_{1} \cos \alpha[k s+d \dot{s}]}{m_{1} m_{2}+m_{2}^{2} \sin ^{2} \alpha}+\frac{\sin ^{2} \alpha}{m_{1}+m_{2} \sin ^{2} \alpha} F, \\
\ddot{s}=-\frac{\left(m_{1}+m_{2}\right)[k s+d \dot{s}]}{m_{1} m_{2}+m_{2}^{2} \sin ^{2} \alpha}-\frac{\cos \alpha}{m_{1}+m_{2} \sin ^{2} \alpha} F .
\end{gathered}
$$

Based on this description of the system dynamics different occurring phenomena are discussed, whereby four different cases are distinct.

Case 1: the relative motion of the mass occurs in vertical direction, i.e. $\alpha=90^{\circ}$, see Fig 5a. In this case the system output is identical to the car position $y=x$ and (Eq. 30) reduces to $P=\left(m_{1}+m_{2}\right)^{-1} \neq 0$. Thus, the equations of motion Eq. (26) and Eqs. (31)-(32) coincide and provide

$$
\begin{aligned}
\left(m_{1}+m_{2}\right) \ddot{y} & =F \\
m_{2} \ddot{s} & =-k s-d \dot{s} .
\end{aligned}
$$

Both equations are here fully decoupled. The force $F$ is orthogonal to the constraint manifold, which is described by the servo-constraint (Eq. 28), see Fig. 5a. This is identical to classical geometric constraints and the control force regulates directly the output. The control action which is necessary to reproduce the output trajectory follows from Eq. (33) as $F_{d}=\left(m_{1}+m_{2}\right) \ddot{y}_{d}$. The dynamics of the mass in vertical direction is described by Eq. (34) and is not influenced by the control force and vice versa. This dynamics of the mass cannot be observed by the system output $y$ and thus in reference to nonlinear control theory this dynamics (Eq. 34) is called internal dynamics, see Isidori (1995) and Sastry (1999).

Case 2: the mass moves along a tilted slope with $0^{\circ}<\alpha<90^{\circ}$, see Fig. 5b. The system dynamics in new coordinates are given by Eqs. (31)-(32) and from Eq. (30) follows $P \neq 0$. This indicates a non-ideal orthogonal realization since the control force has an orthogonal component to the constraint manifold. The control force $F$ can still regulate directly the constraint condition, however it also has a component in tangential direction influencing the relative motion of the mass, see Fig. 5b.

For a given output trajectory $y_{d}$ Eq. (31) can be solved algebraically for the desired control input,

$F_{d}=\frac{m_{1}+m_{2} \sin ^{2} \alpha}{\sin ^{2} \alpha} \ddot{y}_{d}+\frac{m_{1} \cos \alpha[k s+d \dot{s}]}{m_{2} \sin ^{2} \alpha}$.

The control input depends on the second derivative of the system output $\ddot{y}_{d}$ and the unknown states $s, \dot{s}$ which must be computed from Eq. (32). Replacing in Eq. (32) the control input by Eq. (35) yields after reordering

$m_{2} \sin ^{2} \alpha \ddot{s}+d \dot{s}+k s=-\ddot{y}_{d} m_{2} \cos \alpha$.

This is the dynamics of the mass $m_{2}$ on the tilted slope under the applied trajectory tracking control force $F$. In accordance to nonlinear control theory it forms again the internal dynamics. The control force $F$, and thus the system output $y$, influence the internal dynamics, while internal dynamics again influence the control force $F$. In this case 


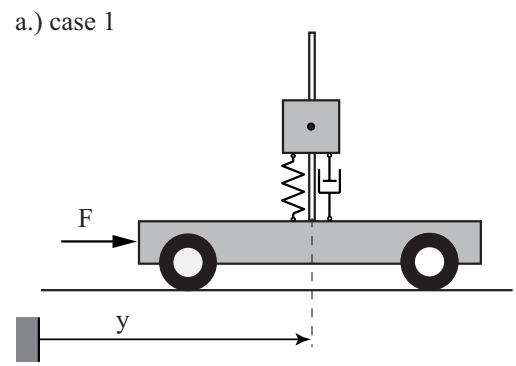

c.) case 3 and 4

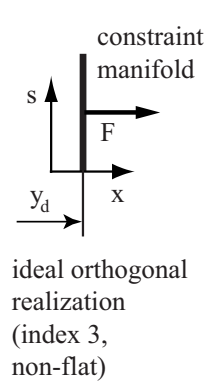

b.) case 2
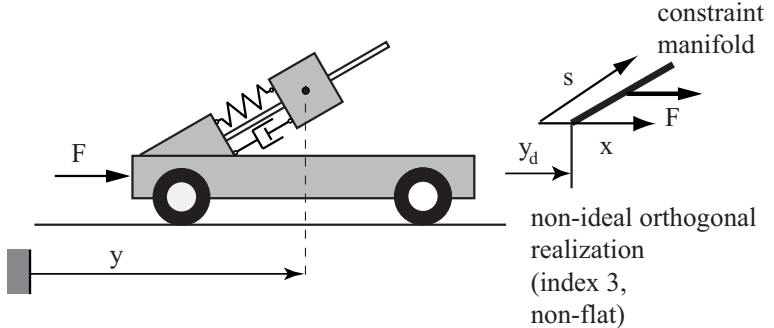
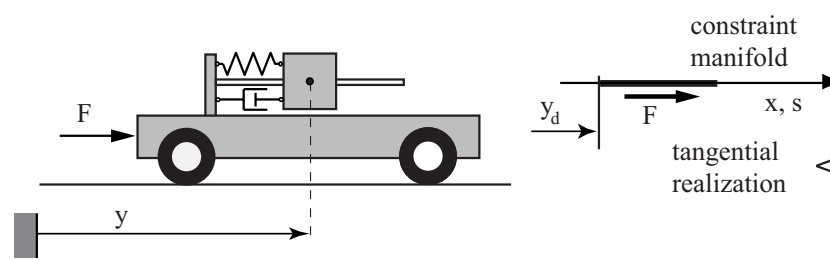

$<$

case 3: with damping: index 4 (non-flat) case 4: without damping: index 5 (differentially flat)

Figure 5. Possible situations arising in servo-constraint problems.

the internal dynamics is a second order differential equation, which is driven by the second derivative of the desired system output trajectory $y_{d}$.

Case 3: the mass moves in horizontal direction, i.e. $\alpha=0^{\circ}$, see Fig. $5 \mathrm{c}$. For this case the equations of motion in new coordinate follow from Eqs. (31)-(32) after reordering as

$m_{2} \ddot{y}=-k s-d \dot{s}$,

$m_{1} m_{2} \ddot{s}=-\left(m_{1}+m_{2}\right)[k s+d \dot{s}]-m_{2} F$.

In addition, it follows from Eq. (30) that $P=0$. This indicates a tangential realization, where the control force $F$ is tangential to the constraint manifold, see Fig. 5c. Thus $F$ cannot directly regulate the servo-constraint, i.e. the system output $y$. This is also seen from Eq. (37), which contains $\ddot{y}$, but not any more the control input $F$. However, output tracking of $y$ is still possible due to coupling with other forces of the system, here the spring force and damper force.

For tracking of the desired output trajectory $y_{d}$, the necessary control input can be computed form Eq. (38) as

$F_{d}=-\frac{\left(m_{1}+m_{2}\right)}{m_{2}}[k s+d \dot{s}]-m_{1} \ddot{s}$.

Firstly, the values of $s, \dot{s}, \ddot{s}$ are unknown. For given $y_{d}$ these can be computed from Eq. (37) as,

$k s+d \dot{s}=-m_{2} \ddot{y}_{d}$.

This is a differential equation for $s$ and poses in this case the internal dynamics. In contrast to the previous two cases, the internal dynamics is here a first order differential equation. For given $\ddot{y}_{d}$ the solution of the internal dynamics Eq. (40) provides the corresponding values $s_{d}$. Then, the values $\dot{s}_{d}$ follow directly form the algebraic solution of Eq. (40) as

$\dot{s}_{d}=-\frac{k s_{d}}{d}-\frac{m_{2}}{d} \ddot{y}_{d}$.

Taking one time-derivative of Eq. (41) yields an algebraic expression for $\ddot{s}_{d}$,

$\ddot{s}_{d}=-\frac{k}{d} \dot{s}_{d}-\frac{m_{2}}{d} y_{d}^{(\mathrm{III})}$.

Thus, all quantities for evaluating the control force $F$ using Eq. (39) are available. The last equation shows, that in contrast to the previous two cases the third derivative of the desired output trajectory $y_{d}$ must be available.

Case 4: the mass moves in horizontal direction and no damping is present, i.e. $\alpha=0^{\circ}$ and $d=0$. Similar to case 3 a tangential realization exists since $P=0$, and the same interpretations apply. The equations of motion in new coordinates simplifies to

$m_{2} \ddot{y}=-k s$,

$m_{1} m_{2} \ddot{s}=-\left(m_{1}+m_{2}\right) k s-m_{2} F$.

Similarly to case 3 , the control force $F_{d}$ can be computed algebraically form Eq. (44), where $s, \ddot{s}$ are unknowns. In contrast to case 3 Eq. (43) is now an algebraic expression for computing $s_{d}$ for given $\ddot{y}_{d}$,

$s_{d}=-\frac{m_{2}}{k} \ddot{y}_{d}$.

Taking two time-derivatives of Eq. (45) yields

$\ddot{s}_{d}=-\frac{m_{2}}{k} y_{d}^{(\mathrm{IV})}$. 
The last equation shows, that in this case the forth derivative of the output trajectory is necessary. Combing Eqs. (44)-(46) yields the control force

$F_{d}=\left(m_{1}+m_{2}\right) \ddot{y}_{d}+\frac{m_{1} m_{2}}{k} y_{d}^{(\mathrm{IV})}$.

Thus, in this case the control input $\boldsymbol{u}_{d}$ and all states $y_{d}, \dot{y}_{d}, s_{d}, \dot{s}_{d}$ of the system can be computed by purely algebraic manipulations, without the need of solving any differential equations. Thus, since all these quantities are specified by the system output $y$ and its four time-derivatives, this case poses a differentially flat system.

Summary and comparison of cases: the servo-constraint problem for this illustrative example has been analyzed using the coordinate transformation approach. Of course, also the DAEs (Eqs. 8-9) or the projected DAEs (Eqs. 15-18) can be established. Thereby, the previous analysis can be used to analyze the differentiation index. In accordance with the discussion at the end of Sect. 2.2 it can be obtained that the differentiation index of the original DAEs (Eqs. 8-9) is one higher than the highest derivative of the system output $y$ which is necessary to compute the control force $F$.

The cases 1 and 2 are orthogonal realizations and therefore provide DAEs with differentiation index 3 , similar to systems with geometric constraints. This is irrespectively of the existence of damping in the system. The dynamics along the constraint manifold is not specified by the output, which forms the internal dynamics. Thus, these are two differentially nonflat mechanical systems. These cases 3 and 4, with tangential realizations, yield higher differentiation index, which is dependent on the existence of damping. In case 3, where damping is present, the system has index 4 . Internal dynamics remain, which in this case is a dynamical system of first order. Thus this example with damping poses a tangential realization for a differentially non-flat system. In case 4 no damping exists and differentiation index 5 arises. Then, the complete motion is specified by the trajectory of the output $y$ and its time-derivatives. No internal dynamics remains and this tangential realization represents the case of a differentially flat underactuated mechanical system. It should be mentioned, that differentially flat systems with higher index exist. Such an example is the $n$-mass-spring chain as analyzed in Blajer (1997b).

This example is representative for the different possible phenomena in servo-constraint problems of underactuated multibody systems. An orthogonal realization yields index 3 and internal dynamics remains, which are described by $f-m$ differential equations of second order. For tangential realization higher index arise. Thereby an increasing differentiation index indicates a reduced size of the internal dynamics and the need for higher derivatives of the output trajectory. In the limit cases no internal dynamics remains, and the system can be inverted purely algebraically, i.e. the system is differentially flat.

\section{Stability of the internal dynamics}

The previous discussion highlights that the inverse model might be a dynamical system, namely containing internal dynamics. This can occur in both cases, the orthogonal realization and the tangential realization. In the computation of the inverse model these internal dynamics must be solved. Thereby, the stability of the internal dynamics, i.e. the system dynamics of the servo-constraint problem, must be investigated carefully. For an ideal orthogonal realization, e.g. classical constraints, the stability properties and analysis of multibody systems with classical constraints applies. This ideal orthogonal realization also occurs in underactuated multibody systems with collocated inputs and outputs, i.e. there is a control input at each system output. This occurs for example in case 1 of the mass on car example which is presented in Sect. 3.

For non-ideal orthogonal and tangential realization, the internal dynamics might be more complex, and stability might not be ensured. This is due to the combination of the multibody system with a control, whereby inputs and outputs are not collocated. In the case of unstable internal dynamics, forward time integration of the internal dynamics might yield unbounded states and control inputs, which provides an unfeasible inverse model. Therefore, careful stability analysis of the internal dynamics is necessary. Using the coordinate transformation approach the internal dynamics of the inverse model can be extracted explicitly, which is helpful in system analysis. For example, for the orthogonal realization the internal dynamics is given by Eq. (25).

In general, the internal dynamics is nonlinear and driven by the desired output trajectory $\boldsymbol{y}_{d}(t)$, posing a nonlinear time-variant system. Since stability analysis of such systems is quite complex, one uses often the concept of zero dynamics, see Isidori (1995); Sastry (1999). The zero dynamics is the internal dynamics under a constant system output, e.g. $\boldsymbol{y}_{d}=\mathbf{0}, \forall t$. This reduces the internal dynamics to a time-invariant nonlinear system. For the orthogonal realization follows from Eq. (25) the zero dynamics,

$\ddot{\boldsymbol{q}}_{\mathrm{u}}=\overline{\boldsymbol{a}}\left(\boldsymbol{q}_{\mathrm{u}}, \dot{\boldsymbol{q}}_{u}\right)$.

Local stability of the zero dynamics can then be checked, e.g. using Lyapunov's indirect method. Local asymptotic stability requires that the linearized zero dynamics has only eigenvalues with negative real part. If at least one eigenvalue has a positive real part the system is unstable. If there are both, eigenvalues with negative real part and purely imaginary eigenvalues, then Lyapunov's indirect method is inconclusive. In this case stability can be checked e.g. using Lyapunov's direct method, see e.g. Khalil (2002), or the center manifold method, see e.g. Isidori (1995).

For a linear system it can be shown that the eigenvalues of the zero dynamics are precisely the zeros of the transfer function, see Isidori (1995). Further details about the close connection between the concept of zero dynamics of 


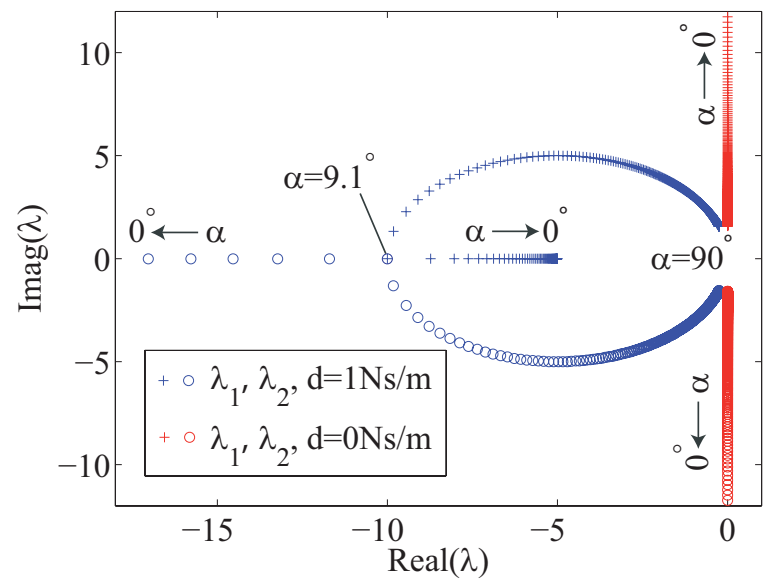

Figure 6. Eigenvalues of zero dynamics for mass on car system.

nonlinear systems and zeros of linear systems are discussed in Isidori and Moog (1988). A linear system is called minimum phase, if all its zeros are in the open left half plane, i.e. its zero dynamics is exponentially stable. This definition is also extended to nonlinear systems. In nonlinear control theory systems with asymptotical stable zero dynamics are called asymptotically minimum phase, otherwise nonminimum phase, see Isidori (1995). It is important to notice, that local asymptotic stability of the zero dynamics is a necessary but not sufficient condition for stability of the driven internal dynamics. This last step is often very complex and from a practical point one restricts often to verify local asymptotic stability of the zero dynamics.

\subsection{Illustrative example 1: stability of mass on car}

For the mass on car example in Sect. 3 internal dynamics remains for cases 1-3. Figure 6 shows the location of the eigenvalues of the zero dynamics for $0^{\circ} \leq \alpha \leq 90^{\circ}$, i.e. case 1 and 2 . The system properties are summarized in Table 1 . The eigenvalues of the zero dynamics follow from the internal dynamics (Eq. 36) with $\ddot{y}_{d}=0$. The internal dynamics is in these cases similar to a spring-mass system and is therefore asymptotically stable for the damped case and stable for the undamped case. For $\alpha=90^{\circ}$ it is identical to a free damped mass-spring system and mass $m_{2}$ vibrates freely in vertical direction. Hereby, in the undamped case the eigenfrequency of the free vibration is $\omega=0.25 \mathrm{~Hz}$. However, with the inclination angle $\alpha$ the dynamic behavior of the internal dynamics changes. Thus for example, for $\alpha=5^{\circ}$ and $d=0 \mathrm{Nsm}^{-1}$ the eigenfrequency of the zero dynamics increases to $2.88 \mathrm{~Hz}$. Also in the damped case the behavior changes dramatically, and for $\alpha<9.1^{\circ}$ and $d=1 \mathrm{Ns} \mathrm{m}^{-1}$ an over-damped behavior occurs. Thus, due to the servo-constraint, the dynamical behavior of the internal dynamics can be quite different from the dynamics of the uncontrolled underactuated multibody system.
Table 1. Properties for mass on car system.

$m_{1}=1 \mathrm{~kg} \quad m_{2}=2 \mathrm{~kg} \quad k=5 \mathrm{~N} \mathrm{~m}^{-1} \quad d=0 \mathrm{Ns} \mathrm{m}^{-1}$ and $d=1 \mathrm{Ns} \mathrm{m}^{-1}$

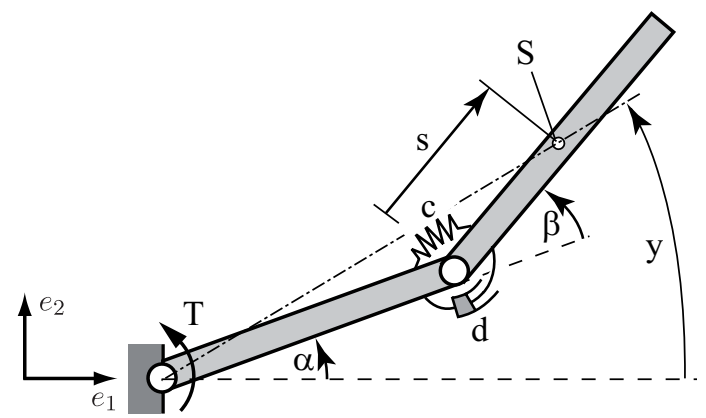

Figure 7. Rotational arm with passive joint.

\subsection{Illustrative example 2: rotational manipulator arm}

As simple example for an underactuated multibody system with unstable internal dynamics a single rotational manipulator arm with a passive joint is considered, see Fig. 7. The rotational arm consists of two identical homogenous links with length $l$, center of mass $s=l / 2$, mass $m$ and inertia $I=m l^{2} / 12$. The first link is actuated by the control torque $u=T$. The second link is connected by a passive joint to the first link, which is supported by a linear spring-damper combination with spring constant $c$ and damper coefficient $d$. Thus, the passive joint manipulator has its elasticity parallel to the joint and shows very many similar properties as manipulators with flexible links, see Seifried et al. (2013). In contrast, such a passive joint system is quite different to a so-called flexible joint manipulator with drive train elasticities, where the flexibility is located between a link of the manipulator and its motor, see De Luca (1998).

The rotational manipulator arm is described by the generalized coordinates $\boldsymbol{q}=[\alpha, \beta]$, whereby $q_{\mathrm{u}}=\beta$ denotes the unactuated coordinate. The arm moves perpendicular to the direction of gravity and the equations of motion are given by

$$
\begin{aligned}
l^{2} m\left[\begin{array}{cc}
\frac{5}{3}+\cos \beta & \frac{1}{3}+\frac{1}{2} \cos \beta \\
\frac{1}{3}+\frac{1}{2} \cos \beta & \frac{1}{3}
\end{array}\right]\left[\begin{array}{c}
\ddot{\alpha} \\
\ddot{\beta}
\end{array}\right] & + \\
{\left[\begin{array}{c}
-0,5 l^{2} m \dot{\beta}(2 \dot{\alpha}+\dot{\beta}) \sin \beta \\
c \beta+d \dot{\beta}+0,5 l^{2} m \dot{\alpha}^{2} \sin \beta
\end{array}\right] } & =\left[\begin{array}{l}
T \\
0
\end{array}\right] .
\end{aligned}
$$

The position of a point $S$ on the second link is described in the body fixed coordinate system by $0<s<l$, see Fig. 7 . The control goal is the tracking of the position of point $S$. For small $\beta$ the position can be described approximately by the system output

$y=\alpha+\frac{s}{s+l} \beta$.

This system output is a linear combination of the two generalized coordinates and can be seen as an auxiliary angle $y$, 
see Fig. 7. For example, with this auxiliary angle the position in $e_{2}$ direction is

$r_{s 2}=l \sin \alpha+s \sin (\alpha+\beta) \approx(l+s) \sin y$.

This approximation holds for small $\beta$, which can be verified by computing the Jacobian linearization around $\beta=0$, see Seifried (2012b). It is noted, that the following computations and analysis for the exact position of $S$ follow the same steps and yield the same results. However, the use of the linearly combined system output (Eq. 50) simplifies the expressions significantly and allows an easier discussion of the results. Similar linearly combined outputs are also often used in end-effector tracking of flexible manipulators, see e.g. De Luca (1998); Seifried et al. (2011).

For this rotational arm the servo-constraint is given by

$c(\boldsymbol{q}, t)=\alpha+\frac{s}{s+l} \beta-y_{d}(t)$

and its Jacobian matrix is

$\boldsymbol{H}=\left[\begin{array}{cc}1 & \frac{s}{l+s}\end{array}\right]$.

With this matrix follows from Eq. (18)

$P=\boldsymbol{H} \boldsymbol{M}^{-1} \boldsymbol{B}=\frac{2 l(3 s \cos \beta-2 l)}{m l^{2}(l+s)(9 \cos (2 \beta)-23)}$.

For $s^{*}=2 l /(3 \cos \beta)$ it is $P=0$, i.e. only for this cases a tangential realization occurs. For small angles $\beta$ the position $s^{*}$ approximates the center of percussion. In Fliess et al. (1995) it is shown, that for a manipulator with one passive joint the center of percussion might yield a differentially flat system output. In contrast, in the following the case $s \neq s^{*}$ is considered, i.e. the case of an orthogonal realization. If $s=0$ then the input and output are collocated since $y=\alpha$. In this case an ideal orthogonal realization occurs, otherwise a non-ideal orthogonal realization exists. From the previous discussions it is obvious that internal dynamics remain.

To analyze the internal dynamics and the servo-constraint problem in more detail, the coordinate transformation approach presented in Sect. 2.4 is applied. The new set of coordinates containing the output are chosen as $\overline{\boldsymbol{q}}=[y, \beta]$. The system dynamics in new coordinates follows from evaluating Eqs. (22)-(23). For a given output trajectory $y_{d}$ the required control input $u_{d}$ follows from Eq. (24). Then, the system dynamics of the inverse model, i.e. the internal dynamics, are described by Eq. (25). For its analysis the zero dynamics is derived, which follows from the internal dynamics with $y=0, \forall t$. For this example the zero dynamics turns out to be

$$
\begin{aligned}
& m l^{2}(s+l)(2 l-3 s \cos \beta) \ddot{\beta} \\
& \quad=-3 m s^{2} l^{2} \sin \beta \dot{\beta}^{2}-6(s+l)^{2}(c \beta+d \dot{\beta}) .
\end{aligned}
$$

For analysis the zero dynamics (Eq. 55) is linearized around its equilibrium point $\beta=0$ yielding

$$
\underbrace{m l^{2}(2 l-3 s)}_{a_{2}} \ddot{\tilde{\beta}}+\underbrace{6 d(s+l)}_{a_{1}} \tilde{\beta}+\underbrace{6 c(s+l)}_{a_{0}} \tilde{\tilde{\beta}}=0 .
$$

Table 2. Properties for rotational manipulator arm.

$$
l=1 \mathrm{~m} \quad m=3.438 \mathrm{~kg} \quad k=50 \mathrm{Nm} \mathrm{rad}^{-1} \quad d=0.25 \mathrm{Nms} \mathrm{rad}^{-1}
$$

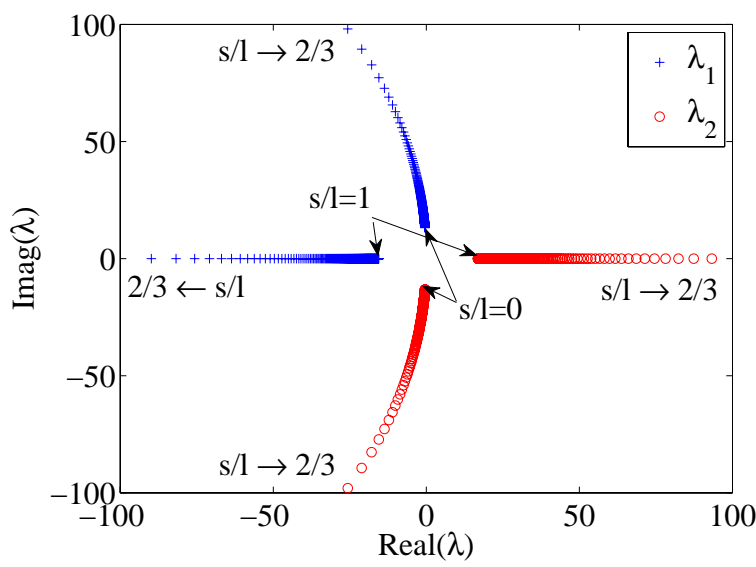

Figure 8. Eigenvalues of zero dynamics for rotational arm.

Thereby $a_{2}, a_{1}, a_{0}$ correspond to the coefficients of the characteristic polynomial. Using Stodola's criterion the linearized zero dynamics (Eq. 56) of the rotational arm is only asymptotically stable, if all coefficients $a_{2}, a_{1}, a_{0}$ have the same sign and are non-zero. The constants $c, d$ of the springdamper combination and the dimensions $l, s$ are by nature positive, yielding positive constants $a_{0}, a_{1}$. Thus also the coefficient $a_{2}$ must be positive to obtain stable internal dynamics. From this follows that for $s / l<2 / 3$ the internal dynamics is locally asymptotically stable, while for $s / l>2 / 3$ the internal dynamics is unstable. The location of the eigenvalues for different $0 \leq s \leq 1$ is shown in Fig. 8. The used system parameters are summarized in Table 2 . This shows clearly that the dynamics of a servo-constraint problem, namely its internal dynamics, might be fundamentally different from the dynamics of the uncontrolled mechanical system. Endeffector tracking, i.e. $s=l$, is in robotic manipulator applications the most interesting case. The presented analysis shows that in this example unstable internal dynamics occur in endeffector trajectory tracking.

The analysis of the rotational arm shows that the stability of the internal dynamics can depend on the choice of the system output location. In addition, if a non-homogenous design for the links is admitted, the stability of the internal dynamics also depends on the mass distribution of the links, see the analysis in Seifried (2012b). Then, the mass distribution can also be designed in such a way that for end-effector trajectory tracking stable internal dynamics remain.

The presented analysis and the discussed structural properties for this small servo-constraint system are representative for many multibody systems with passive joints and flexible manipulators in end-effector trajectory tracking. Analysis of 
such systems are given e.g. in Seifried (2012b) and Seifried et al. (2011), respectively.

\section{Numerical solution}

In most cases the inverse model requires a numerical solution, whereby the solution method depends on the previously analyzed system properties. In the following some basic numerical solution methods are summarized for differentially flat systems, systems with stable internal dynamics and systems with unstable internal dynamics. For demonstration purpose these methods are applied to the two presented illustrative examples. The following presentation highlights some solution issues and demonstrates also the effect of the different system properties. However, it is not meant as a full in depth investigation of numerical time integrators for servoconstraint problems.

\subsection{Differentially flat systems}

For differentially flat systems, a purely algebraic inverse model can be derived. However, this requires often a large number of symbolic time-derivations and manipulations of the output equation and the equations of motion. This might be possible for small systems, such as in case 4 of the mass on car system, but for larger systems these symbolic computations might become very burdensome. Therefore, also for differentially flat systems a numerical solution based on the servo-constraint approach might be useful.

Due to the tangential realization in underactuated differentially flat systems the original servo-constraint formulation (Eqs. 8-9) has differentiation index greater 3. For the numerical solution the use of the projection approach presented in Sect. 2.3 might be advantageous, yielding a index reduction. Then, the set of differential-algebraic Eqs. (15)(18) must be solved numerically. For readability these equations are summarized as

$$
\begin{aligned}
\dot{\boldsymbol{q}} & =\boldsymbol{v} \\
\boldsymbol{A} \dot{\boldsymbol{v}} & =\boldsymbol{a}(\boldsymbol{v}, \boldsymbol{q}, \boldsymbol{u}) \\
\mathbf{0} & =\boldsymbol{b}\left(\boldsymbol{v}, \boldsymbol{q}, \boldsymbol{u}, \ddot{\boldsymbol{y}}_{d}\right) \\
\mathbf{0} & =\boldsymbol{c}\left(\boldsymbol{q}, \boldsymbol{y}_{d}\right) .
\end{aligned}
$$

The solution of this set of $2 f+m$ equations are variations in time of the $m$ control inputs $\boldsymbol{u}(t)$ which are required for the exact reproduction of the desired output trajectory $\boldsymbol{y}_{d}(t)$, and variations of the $2 f$ states $\boldsymbol{q}(t), \boldsymbol{v}(t)=\dot{\boldsymbol{q}}(t)$ in the specified motion.

Since differentially flat systems can completely be inverted algebraically, the output specifies completely the entire motion of the system and Eqs. (57)-(60) do not contain any internal dynamics. This allows the efficient use of rather simple solution formulas. A simple numerical solution schema for solving the DAEs can be based on the Euler backward differentiation scheme, as proposed by Blajer and Kolodziejczyk

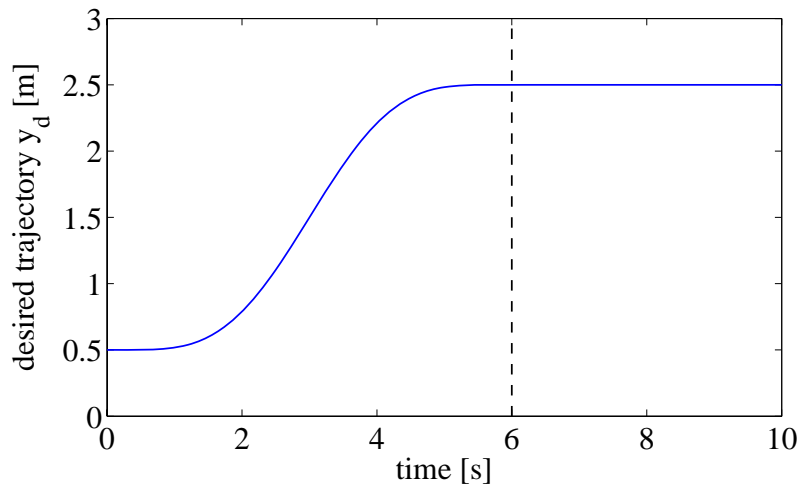

Figure 9. Desired output trajectory $y_{d}$ for mass $m_{2}$.

(2004). Thereby the time derivatives $\dot{\boldsymbol{q}}$ and $\dot{\boldsymbol{v}}$ are approximated with their backward differences $\left(\boldsymbol{q}_{n+1}-\boldsymbol{q}_{n}\right) / \Delta t$ and $\left(\boldsymbol{v}_{n+1}-\boldsymbol{v}_{n}\right) / \Delta t$, respectively. Thereby $\Delta t$ is the constant integration time step, such that $t_{n+1}=t_{n}+\Delta t$. With the known values $\boldsymbol{q}_{n}, \boldsymbol{v}_{n}$ at time $t_{n}$, the solution $\boldsymbol{q}_{n+1}, \boldsymbol{v}_{n+1}, \boldsymbol{u}_{n+1}$ at time $t_{n+1}$ can be obtained from the solution to the set of nonlinear algebraic equations

$$
\begin{aligned}
\boldsymbol{q}_{n+1}-\boldsymbol{q}_{n}-\Delta t \boldsymbol{v}_{n+1} & =\mathbf{0} \\
\boldsymbol{A}\left(\boldsymbol{q}_{n+1}\right)\left[\boldsymbol{v}_{n+1}-\boldsymbol{v}_{n}\right]-\Delta t \boldsymbol{a}\left(\boldsymbol{v}_{n+1}, \boldsymbol{q}_{n+1}, \boldsymbol{u}_{n+1}\right) & =\mathbf{0} \\
\boldsymbol{b}\left(\boldsymbol{v}_{n+1}, \boldsymbol{q}_{n+1}, \boldsymbol{u}_{n+1}, \ddot{\boldsymbol{y}}_{d, n+1}\right) & =\mathbf{0} \\
\boldsymbol{c}\left(\boldsymbol{q}_{n+1}, \boldsymbol{y}_{d, n+1}\right) & =\mathbf{0} .
\end{aligned}
$$

This schema can also be used for systems with mixed geometric and servo-constraints as presented in Blajer and Kolodziejczyk (2011).

This very basic solution schema is applied to the mass on car system. Mass 2 should follow the output trajectory

$$
\begin{aligned}
y_{d}=y_{0} & +\left[126\left(\frac{t}{t_{\mathrm{f}}-t_{0}}\right)^{5}-420\left(\frac{t}{t_{\mathrm{f}}-t_{0}}\right)^{6}+540\left(\frac{t}{t_{\mathrm{f}}-t_{0}}\right)^{7}\right. \\
& \left.-315\left(\frac{t}{t_{\mathrm{f}}-t_{0}}\right)^{8}+70\left(\frac{t}{t_{\mathrm{f}}-t_{0}}\right)^{9}\right]\left(y_{\mathrm{f}}-y_{0}\right),
\end{aligned}
$$

whereby starting point $y_{0}=0.5 \mathrm{~m}$ and finial point $y_{\mathrm{f}}=2.5 \mathrm{~m}$ are chosen. After reaching the final point at time $t_{\mathrm{f}}=6 \mathrm{~s}$ the output is at rest. The complete simulation time is $10 \mathrm{~s}$. This trajectory is designed in such a way, that also its higher derivatives are sufficiently smooth. The trajectory is shown in Fig. 9.

It is noticed that the original servo-constraint problem has differentiation index 5 , while the projection approach yields index 3. Figure 10 shows the control forces $u_{d}$ computed with the projection approach and compared to the analytical solution from Eq. (47). This shows, that this rough computational scheme is of acceptable accuracy for appropriately small values of $\Delta t$. Here, the Euler backward schema yields nearly identical control inputs as the analytical solution for time sizes $1 \mathrm{~ms}$ and $10 \mathrm{~ms}$. Only after increasing the time step size to $100 \mathrm{~ms}$ significant errors in form of a time delay are obvious. For differentially flat systems, the inverse model is 


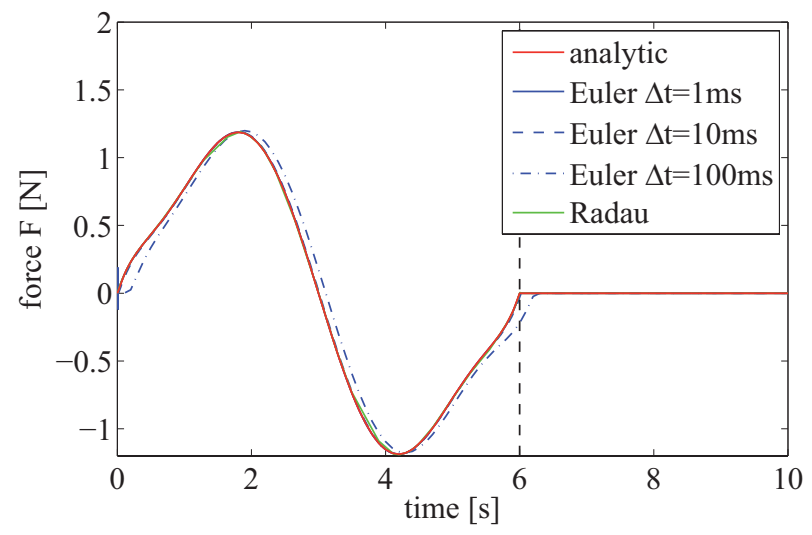

Figure 10. Mass on car - case 4, flat system: computed control input $\boldsymbol{u}_{d}$.

completly specified by the output. Thus, it can be argued that in this case the issue of numerical damping, which for such a simple discretization schema normally appears, might not pose a significant problem here.

More accurate time discretization schema can be used for the solution of the servo-constraint problem of differentially flat underactuated multibody systems. Therefore, in Fig. 10 also the solution obtained by a Radau IIa schema is added. This method is capable of handling index 3 DAEs, see Hairer and Wanner (2010). Hereby an accurate solution is obtained by using only 56 time points. This compares to $1000-10000$ points using the Euler backward schema. In literature further solution methods have been proposed for differentially flat servo-constraint problems. Betsch et al. (2007, 2008) use a energy conserving schema for solving the projected Eqs. (57)-(60), whereby redundant coordinates are used. For a differentially flat crane Fumagalli et al. (2011) propose a solution based on backward differentiation formula.

In Fig. 11 the analytical solution for the desired trajectories of the generalized coordinates and its derivatives are shown. Since hardly any differences to the numerical solutions are visible, the presentation of the numerical solutions are omitted here. From Fig. 11, as well as from the control force plot, it is seen that the complete system is at rest after $t_{\mathrm{f}}=6 \mathrm{~s}$, indicating the final position of the output. This is due to the fact that for this system the output trajectory specifies the complete system behavior and no internal dynamics remain. This is typical for a differentially flat system.

\subsection{Systems with stable internal dynamics}

Underactuated multibody systems with stable internal dynamics can be solved by forward time integration. Therefore, the same numerical integrators as in the previous discussed differentially flat case might be used. This is demonstrated using the mass on car system with an inclination angle of $\alpha=5^{\circ}$, representing a non-ideal orthogonal realiza-
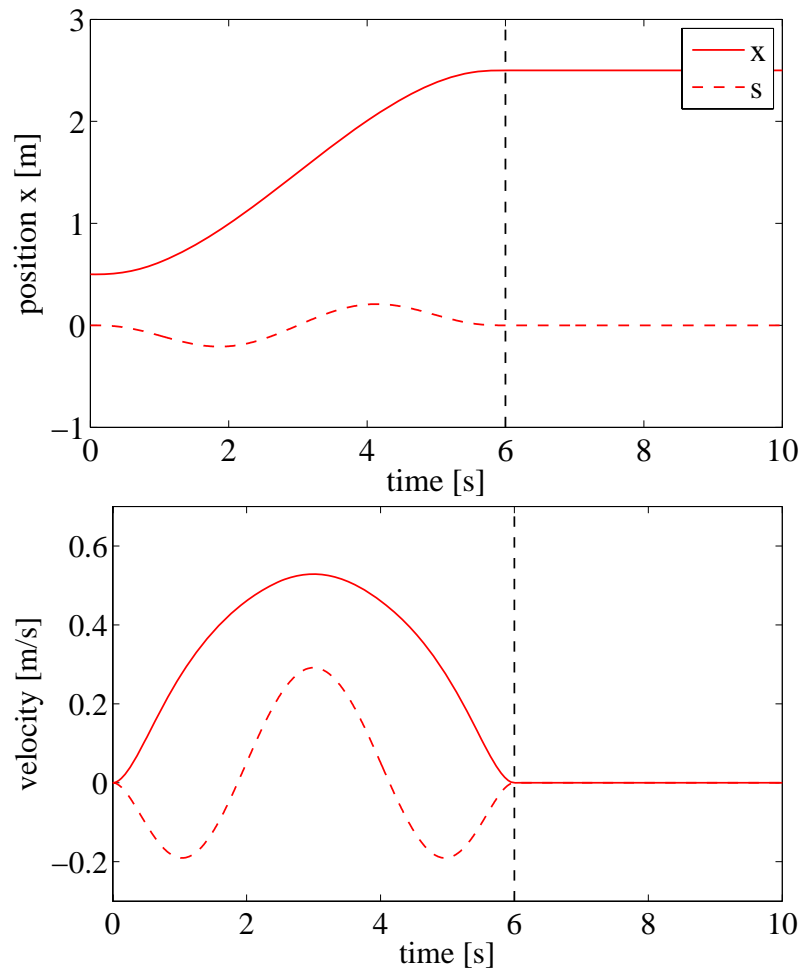

Figure 11. Mass on car - case 4, flat system: computed states $\boldsymbol{q}_{d}, \dot{\boldsymbol{q}}_{d}$

tion. Thereby the case of a strongly damped system with $d=1 \mathrm{Nsm}^{-1}$ and the undamped system are considered.

By using the projection approach, index 1 DAEs arise for the orthogonal realization. As numerical solution schema for the projected servo-constraint Eqs. (15)-(18) the previously presented Euler backward schema with time step size $1 \mathrm{~ms}$ and $10 \mathrm{~ms}$ are used. In addition a numerical backward differentiation formula is used, as implemented in the Matlab function ode15s, see Shampine et al. (1999). This is capable of solving index 1 DAEs. Also the coordinate transformation approach is used. Hereby the internal dynamics is given explicitly by Eq. (36) and the control input follows from Eq. (35). For the numerical solution of the internal dynamics (Eq. 36) the Matlab ode45 integrator is used, which is an explicit Runge-Kutta formula of order 4 and 5 using the Dormand-Prince pair.

The obtained control forces using the different solution methods are shown for the damped and undamped case in Fig. 12. The velocities of the generalized coordinates are presented in Fig. 13. Since differences between the solution methods are seen best in the control force, the velocity plot shows only the solution obtained using the coordinate transformation approach.

Due to the presence of the internal dynamics the output does not specify completely the motion of the system. After the output reaches its final position, it remains at rest. However, the system itself is at time $t_{\mathrm{f}}=6 \mathrm{~s}$ not at rest, as 

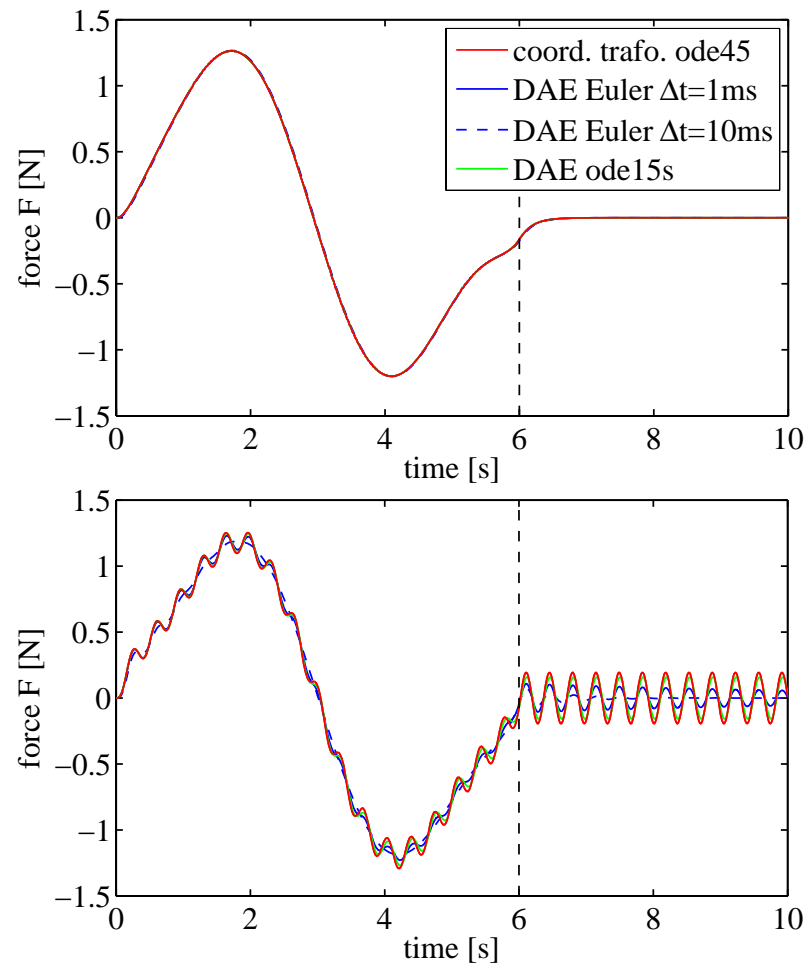

Figure 12. Mass on car - case 2, $\alpha=5^{\circ}$ : computed control input $\boldsymbol{u}_{d}$. With damping $d=1 \mathrm{Ns} \mathrm{m}^{-1}$ (top) and without damping (bottom).

seen from the force and velocity plots. The remaining motion of the system is its zero dynamics. For the case with $d=1 \mathrm{Nsm}^{-1}$ an overdamped internal dynamics occurs, as also seen from the eigenvalue plot of the zero dynamics in Fig. 6. Thus, the internal dynamics decays rapidly. In contrast for the undamped case strong vibrations occur. These are best visible in the control force plot, whereby vibrations of the internal dynamics occur during trajectory tracking as well as after the output reaches its final position. It should be noted, that the eigenfrequency of the internal dynamics is much higher than the natural frequency of the uncontrolled system, see also Fig. 6.

For the damped case the numerical solutions using the different methods widely coincide, which is seen in the upper plot of Fig. 12. In contrast, for the undamped case some clear differences are observed, see the lower plot of Fig. 12. Here vibrations occur whose frequency is over 10 times higher than in the uncontrolled case. These high frequency vibrations of the internal dynamics are numerically damped using the Euler backward schema. This yields less accurate control inputs, deteriorating the performance of the feedforward control. However, using the more sophisticated methods, the control inputs computed with the projection approach and the coordinate transformation approach coincide.
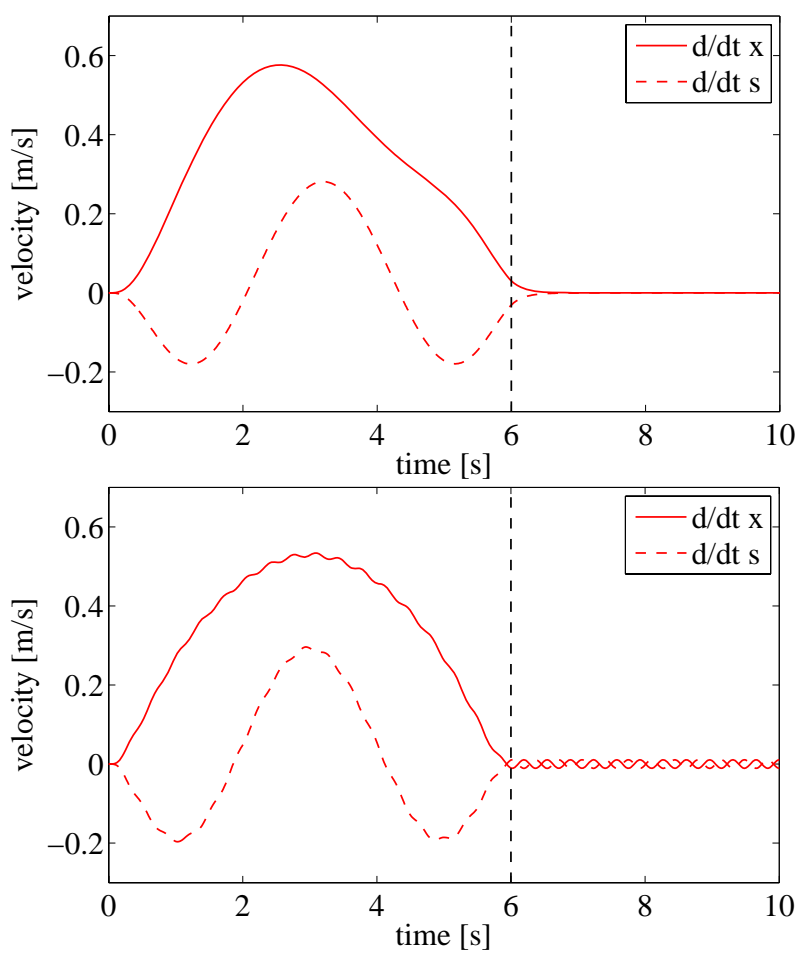

Figure 13. Mass on car - case 2, $\alpha=5^{\circ}$ : generalized velocities $\dot{\boldsymbol{q}}_{d}$ With damping $d=1 \mathrm{Nsm}^{-1}$ (top) and without damping (bottom).

\subsection{Systems with unstable internal dynamics}

Forward time integration of systems with unstable internal dynamics yield unbounded states and thus unbounded control inputs $\boldsymbol{u}_{d}$. This does not provide a feasible feedforward control. Therefore the previously presented solution schema for differentially flat systems and systems with stable internal dynamics cannot be used. Compared to the previous cases, there are much less approaches for the solution of inverse models with unstable internal dynamics.

In the following the so-called stable inversion approach is briefly presented, which is due to Devasia et al. (1996). This approach has been so far applied for solving the internal dynamics given as ODE, such as Eq. (25) for the orthogonal realization. Examples are the feedforward control design of flexible manipulators, see Seifried et al. (2011). With this approach bounded trajectories $\boldsymbol{q}_{\mathrm{u}}, \dot{\boldsymbol{q}}_{u}$ of the internal dynamics (Eq. 25) and thus bounded control inputs $\boldsymbol{u}_{d}$ are obtained. However, the solution might be non-causal, i.e. the trajectories depend on future states providing a so-called pre-actuation phase.

In stable inversion it is assumed that the starting and ending point of the desired system output trajectory $\boldsymbol{y}_{d}$ are equilibrium points of the multibody system. Further, it is required that the corresponding equilibrium points of the internal dynamics are hyperbolic such that stable manifold $\boldsymbol{W}^{\mathrm{s}}$ and unstable manifold $\boldsymbol{W}^{\mathrm{u}}$ exist at each equilibrium point, Sastry 


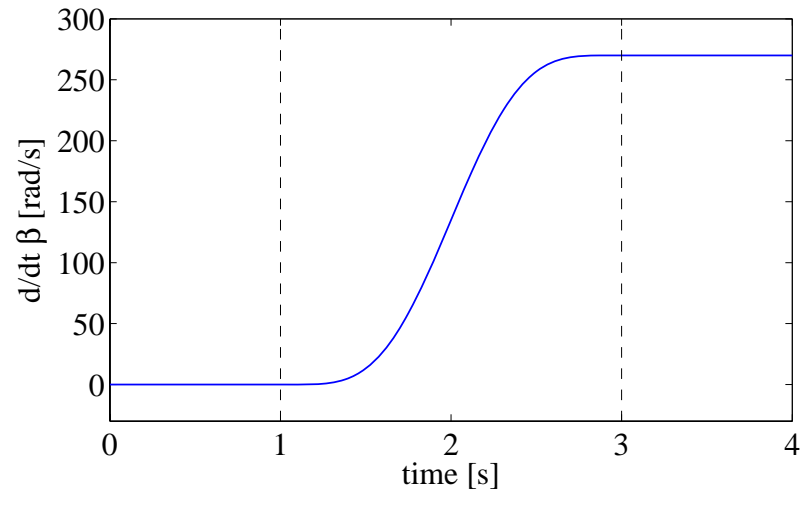

Figure 14. Desired output trajectory $y_{d}$ for rotational manipulator.

(1999). Any trajectory starting on the stable manifold $\boldsymbol{W}^{\mathrm{s}}$ converges to the equilibrium point as time $t \rightarrow \infty$ and any trajectory starting on the unstable manifold $\boldsymbol{W}^{\mathrm{u}}$ converges to the equilibrium point as time $t \rightarrow-\infty$. The solution of the stable inversion is then formulated as a two-sided boundary value problem. The boundary conditions are described by the unstable and stable eigenspaces $\boldsymbol{E}_{0}^{\mathrm{u}}, \boldsymbol{E}_{\mathrm{f}}^{\mathrm{s}}$ at the corresponding equilibrium points, which are local approximations of the unstable manifold $\boldsymbol{W}_{0}^{\mathrm{u}}$ and stable manifold $\boldsymbol{W}_{\mathrm{f}}^{\mathrm{s}}$, respectively, Sastry (1999). This yields for the internal dynamics bounded trajectories $\boldsymbol{q}_{\mathrm{u}}, \dot{\boldsymbol{q}}_{\mathrm{u}}$ which start at time $t_{0}$ on the unstable manifold $\boldsymbol{W}_{0}^{\mathrm{u}}$ and reach the stable manifold $\boldsymbol{W}_{\mathrm{f}}^{\mathrm{s}}$ at time $t_{\mathrm{f}}$. Thus, the initial conditions $\boldsymbol{q}_{u_{0}}, \dot{\boldsymbol{q}}_{u_{0}}$ at time $t_{0}$ cannot exactly be pre-designated. Therefore, a pre-actuation phase is necessary which drives the system along the unstable manifold to a particular initial condition $\boldsymbol{q}_{\mathrm{u}}\left(t_{0}\right), \dot{\boldsymbol{q}}_{\mathrm{u}}\left(t_{0}\right)$, while maintaining the constant output $\boldsymbol{y}_{d}=\boldsymbol{y}_{d}\left(t_{0}\right)$. Also a post-actuation phase is necessary to drive the internal dynamics along the stable manifold close to its resting position. The two-sided boundary value problem must be solved numerically, e.g. by a finite difference method as proposed by Taylor and $\mathrm{Li}$ (2002).

This stable inversion approach is applied to the rotational manipulator example. Thereby, the system output $y$ should follow the trajectory shown in Fig. 14. The system output should be at rest for $t<t_{0}=1 \mathrm{~s}$ and $t>t_{\mathrm{f}}=3 \mathrm{~s}$. For $1 \mathrm{~s} \leq t \leq 3 \mathrm{~s}$ the output should move form $0^{\circ}$ to $270^{\circ}$, whereby the trajectory has the same form as Eq. (65).

For the model inversion the internal dynamics is derived using the coordinate transformation approach, and is given by Eq. (25). Thus for the internal dynamics a second order differential equation for $\beta$ arises, which is the unactuated coordinate of this manipulator arm. The numerical solution of the stable inversion problem is computed in Matlab using the general boundary value solver $b v p 5 c$, see Kierzenka and Shampine (2008). In Fig. 15 the obtained control torque $u_{d}=T$ is shown. Figure 16 presents the trajectory for the unactuated coordinate $\beta$. It is clearly seen that the obtained solution for the inverse model is bounded. However, it turns out, that the computed solution is non-causal, i.e. trajectories

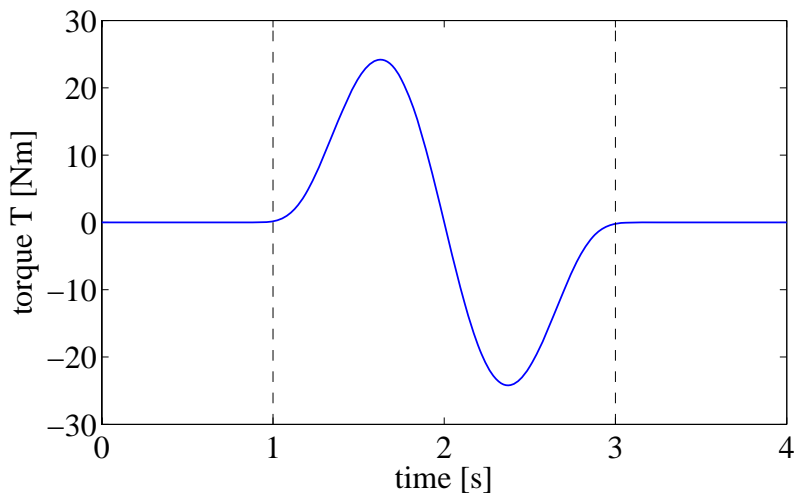

Figure 15. Rotational manipulator arm: computed control input $\boldsymbol{u}_{d}$.
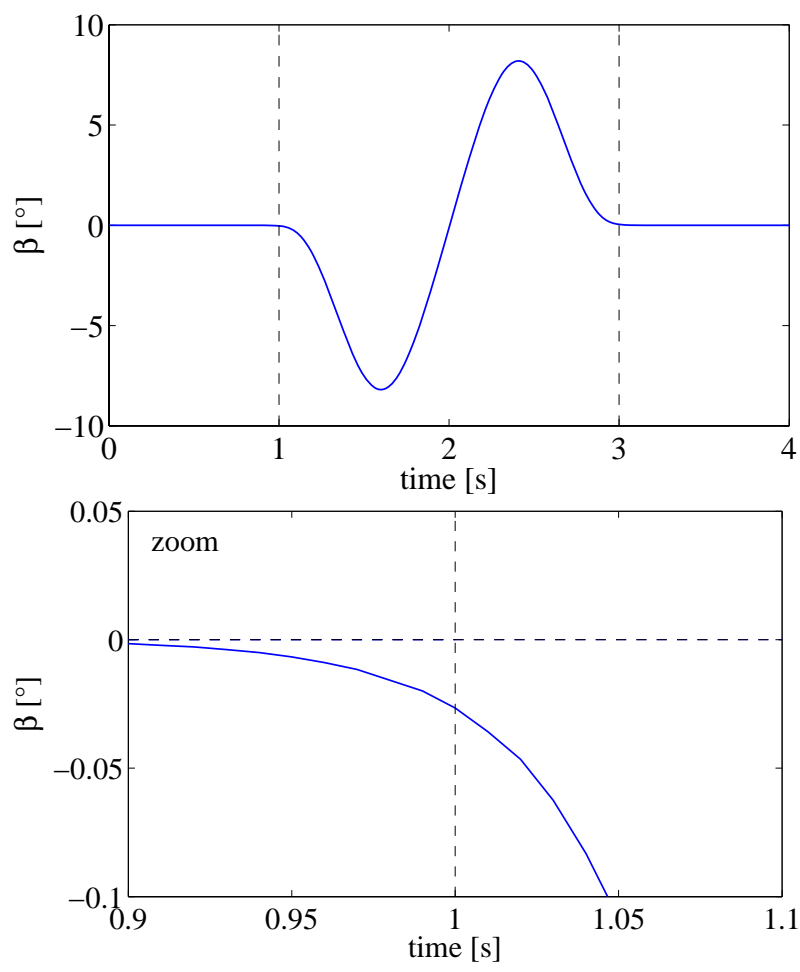

Figure 16. Rotational manipulator arm: computed trajectory of unactuated coordinate $\beta$.

for $\beta$ as well as the control torque $T$ start before $t=1 \mathrm{~s}$, which indicates the start of the output trajectory. This is best seen in the enlargement of $\beta$ around $t=1 \mathrm{~s}$, which is also shown in Fig. 16.

Alternative methods for solving model inversion for systems with unstable internal dynamics have been recently proposed for the original servo-constraint formulation (Eqs. 8 9). In Seifried et al. (2013) the problem is projected numerically into the unconstrained subspace, from which the internal dynamics as ODE arises. This is then solved using the previously described stable inversion method. This approach has been applied to the feedforward control of a flexible 
multibody system with a kinematic loop, featuring 18 unactuated elastic degrees of freedom which represent the internal dynamics. Other solution approaches have been proposed, where the servo-constraint problem is reformulated into optimization problems, see Moberg and Hanssen (2009); Bastos et al. (2011). For example, in Bastos et al. (2011) the solution of the servo-constraint problem is stated as optimal control problem, where the objective function is the minimization of the trajectories of internal dynamics. Thus a bounded solution can be obtained. The stated problem is then solved by standard optimal control algorithms using a direct collocation method.

\section{Conclusions}

The servo-constraint approach is an efficient and straightforward way for deriving inverse models of underactuated multibody systems, yielding firstly a set of DAEs. In the servo-constraint approach the control inputs are used to meet the imposed servo-constraints. In the case of an orthogonal realization the control forces can directly regulate the constraint manifold, which is defined by the servo constraint. This provides a DAE system with differentiation index 3 . In contrast for a tangential realization the control forces can only regulate the constraint manifold indirectly, yielding index larger 3. For the orthogonal realization of underactuated multibody systems internal dynamics remain in the inverse model. For a tangential realization, depending on the system parameters, internal dynamics might or might not exist. In the latter case the complete motion of the system is specified by the system output and a finite number of its derivatives. Such a system is called differentially flat. In this paper it is demonstrated that these different system properties, i.e. the existence of internal dynamics, might be changed by altering some system parameters. For the presented mass-springdamper system on a car these system parameters are the inclination angle and the damping parameter.

In order to obtain a meaningful solution these different system properties must be investigated. Especially the internal dynamics might be very different form the dynamics of the system without servo-constraint. In the extreme case the internal dynamics might be unstable. The stability of the internal dynamics depends on physical system parameters and the selected system output. For example, the presented case of a passive joint manipulator shows, that the stability of the internal dynamics changes, if the output location is altered. Here, using the end-effector position of the manipulator as system output yields unstable internal dynamics. This can also be observed for many manipulators with flexible links.

In this paper two formulations have been presented for the analysis and solution of servo-constraint problems. The first approach is a projection approach which yields a DAE formulation with reduced differentiation index. The second approach is a coordinate transformation approach which yields, at least for the orthogonal realization, an ODE formulation. This coordinate transformation approach is inspired by nonlinear control theory. It is especially useful if the servoconstraint equations can be solved analytically. For the numerical solution of inverse models time integration schemas can be used for differentially flat systems or systems with stable internal dynamics. Hereby a variety of time-integration schema for DAEs or ODEs can be used. For the projection approach a simple Euler-backwards differentiation schema might be used. This provides accurate results for flat systems. In contrast, it might provide only rough accuracy for systems with internal dynamics, which is due to errors induced by numerical damping. In this case more sophisticated numerical methods must be used.

For systems with unstable internal dynamics forward time integration cannot be used to compute a meaningful feedforward control. Therefore, the problem can be reformulate into a boundary value problem, which must be solved offline. This provides then a bounded but non-causal solution for the control inputs and the trajectories of all states.

Acknowledgements. The first author would like to thank the German Research Foundation (DFG) for financial support of the project within the Cluster of Excellence in Simulation Technology (EXC 310/1) at the University of Stuttgart.

Edited by: A. Tasora

Reviewed by: two anonymous referees

\section{References}

Bajodah, A., Hodges, H., and Chen, Y.-H.: Inverse Dynamics of Servo-Constraints Based on the Generalized Inverse, Nonlinear Dynam., 39, 179-196, 2005.

Bastos, G., Seifried, R., and Brüls, O.: Inverse Dynamics of Underactuated Multibody Systems using a DAE Optimal Control Approach, in: Proceedings of the ECCOMAS Thematic Conference Multibody Dynamics 2011, edited by: Samin, J. C. and Fisette, P., Brussels, Belgium, 4-7 July 2011.

Betsch, P., Uhlar, S., and Quasem, M.: On the Incorporation of Servo Constraints into a Rotationless Formulation of Flexible Multibody Dynamics, in: Proceedings of the ECCOMAS Thematic Conference Multibody Dynamics, Milano, Italy, 2007.

Betsch, P., Uhlar, S., and Quasem, M.: Numerical Integration of Discrete Mechanical Systems with Mixed Holonomic and Control Constraints, in: Proceedings of the 4th Asian Conference on Multibody Dynamics, Jeju, Korea, 2008.

Blajer, W.: Index of Differential-Algebraic Equations Governing the Dynamics of Constraint Mechanical Systems, Appl. Math. Model., 16, 70-77, 1992.

Blajer, W.: A Geometric Unification of Constrained System Dynamics, Multibody Syst. Dyn., 1, 3-21, 1997a.

Blajer, W.: Dynamics and Control of Mechanical Systems in Partly Specified Motion, J. Frankl. Inst., 334B, 407-426, 1997 b.

Blajer, W.: A Geometric Interpretation and Uniform Matrix Formulation for Multibody System Dynamics, ZAMM, 81, 247-259, 2001. 
Blajer, W. and Kolodziejczyk, K.: A Geometric Approach to Solving Problems of Control Constraints: Theory and a DAE Framework, Multibody Syst. Dyn., 11, 343-364, 2004.

Blajer, W. and Kolodziejczyk, K.: Control of Underactuated Mechanical Systems with Servo-Constraints, Nonlinear Dynam., 50, 781-791, 2007.

Blajer, W. and Kolodziejczyk, K.: Improved DAE Formulation for Inverse Dynamics Simulation of Cranes, Multibody Syst. Dyn., 25, 131-143, 2011.

Blajer, W. and Seifried, R.: From Orthogonal to Tangential Control of Underactuated Mechanical Systems in Partly Specified Motion - A Case Study Illustration, Int. J. Appl. Mech. Eng., 17, 689-706, 2012

Blajer, W., Seifried, R., and Kolodziejczyk, K.: Diversity of ServoConstraint Problems for Underactuated Mechanical Systems: A Case Study Illustration, Solid State Phenomena, Mechatronic Systems and Materials, IV, 473-482, 2013.

Campbell, S.: High-Index Differential Algebraic Equations, Mech. Based Des. Struc., 23, 199-222, 1995.

De Luca, A.: Trajectory Control of Flexible Manipulators, Control Problems in Robotics and Automation, in: Lecture Notes in Control and Information Sciences, edited by: Siciliano, B. and Valavanis, K. P., Springer, London, 230, 83-104, 1998.

Devasia, S., Chen, D., and Paden, B.: Nonlinear Inversion-Based Output Tracking, IEEE T. Automat. Contr., 41, 930-942, 1996.

Fliess, M., Lévine, J., Martin, P., and Rouchon, P.: Flatness and Defect of Nonlinear Systems: Introductory Theory and Examples, Int. J. Control, 61, 1327-1361, 1995.

Frye, J. and Fabien, B.: Control of Constrained Systems Described by Lagrangin DAEs, in: Proceedings of the ASME 2011 International Design Engineering Technical Conferences \& Computers and Information in Engineering Conference, Washington, 28-31 August 2011.

Fumagalli, A., Masarati, P., Morandini, M., and Mantegazza, P.: Control Constraint Realization for Multibody Systems, J. Comput. Nonlin. Dyn., 6, 011002-1-8, 2011.

Hairer, E. and Wanner, G.: Solving Ordinary Differential Equations II - Stiff and Differential Algebraic Problems, Springer, Berlin, 2010.

Isidori, A.: Nonlinear Control Systems, Springer, London, 1995.

Isidori, A. and Moog, C.: On the Nonlinear Equivalent of the Notion of Transmission Zeros, Modelling and Adaptive Control, in: Lecture Notes in Control and Information Sciences, 105, Springer, 1988.

Kierzenka, J. and Shampine, L.: A BVP Solver that Controls Residual and Error, Journal of Numerical Analysis, Industrial and Applied Mathematics, 3, 27-41, 2008.

Khalil, H. K.: Nonlinear Systems, Prentice Hall, Upper Saddle River, 3rd Edn., 2002.

Kirgetov, V. I.: The Motion of Controlled Mechanical Systems with Prescribed Constraints (Servoconstraints), J. Appl. Math. Mech., 21, 433-466, 1967.

Kovács L., Köveces, J., Zelei, A., Bencsik, L., and Stépan, G.: Servo-Constraint Based Computed Torque Control of Underactuated Mechanical Systems, in: Proceedings of the ASME 2011 International Design Engineering Technical Conferences \& Computers and Information in Engineering Conference, Washington, DETC2011-48533, 28-31 August 2011.
Masarati, P., Morandini, M., and Fumagalli, A.: Control Constraint Realization Applied to Underactuated Aerospace Systems, in: Proceedings of the ASME 2011 International Design Engineering Technical Conferences \& Computers and Information in Engineering Conference, Washington, DETC2011-47276, 28-31 August 2011.

Moberg, S. and Hanssen, S.: A DAE Approach to Feedforward Control of Flexible Manipulators, in: Proceedings of the 2007 IEEE International Conference on Robotics and Automation, 34393444, 2007.

Moberg, S. and Hanssen, S.: Inverse Dynamics of Flexible Manipulators, in: Proceedings of the ECCOMAS Thematic Conference Multibody Dynamics, Warsaw, Poland, 2009.

Rosen, A.: Applying the Lagrange Method to Solve Problems of Control Constraints, J. Appl. Mech., 66, 1013-1015, 1999.

Rothfuss, R., Rudolph, J., and Zeitz, M.: Flachheit: Ein neuer Zugang zur Steuerung und Regelung nichtlinearer Systeme, at Automatisierungstechnik, 45, 517-525, 1997 (in German).

Sastry, S.: Nonlinear Systems: Analysis, Stability and Control, Springer, New York, 1999.

Schiehlen, W., Guse, N., and Seifried, R.: Multibody Dynamics in Computational Mechanics and Engineering Applications, Comput. Meth. Appl. M., 195, 5509-5522, 2006.

Seifried, R.: Two Approaches for Feedforward Control and Optimal Design of Underactuated Multibody Systems, Multibody Syst. Dyn., 27, 75-93, 2012a.

Seifried, R.: Integrated Mechanical and Control Design of Underactuated Multibody Systems, Nonlinear Dynam., 67, 1539-1557, $2012 b$.

Seifried, R., Held, A., and Dietmann, F.: Analysis of Feed-Forward Control Design Approaches for Flexible Multibody Systems, Journal of System Design and Dynamics, 5, 429-440, 2011.

Seifried, R., Burkhardt, M., and Held, A.: Trajectory Control of Serial and Parallel Flexible Manipulators using Model Inversion, Multibody Dynamics: Computational Methods and Applications, in: Computational Methods in Applied Sciences, edited by: Samin, J. C. and Fisette, P., Springer, 28, 53-75, 2013.

Shampine, L., Reichelt, M., and Kierzenka, J.: Solving Index-1 DAEs in MATLAB and Simulink, SIAM Rev., 41, 538-552, 1999.

Siciliano, B., Sciavicco, L., Villani, L., and Oriolo, G.: Robotics Modelling, Planning and Control, Springer, London, 2010.

Spong, M., Hutchinson, S., and Vidyasagar M.: Robot Modeling and Control, John Wiley \& Sons, Hoboken, 2006.

Taylor, D. and Li, S.: Stable Inversion of Continuous-Time Nonlinear Systems by Finite-Difference Methods, IEEE T. Automat. Contr., 47, 537-542, 2002. 